\section{A) Check for updates}

Cite this: Nanoscale, 2022, 14, 3324

\title{
Controlling disorder in self-assembled colloidal monolayers via evaporative processes
}

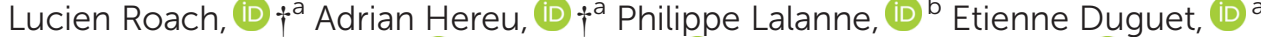 \\ Mona Tréguer-Delapierre, (D) ${ }^{a}$ Kevin Vynck (iD) *c and Glenna L. Drisko (D) *a
}

\begin{abstract}
Monolayers of assembled nano-objects with a controlled degree of disorder hold interest in many optical applications, including photovoltaics, light emission, sensing, and structural coloration. Controlled disorder can be achieved through either top-down or bottom-up approaches, but the latter is more suited to large-scale, low-cost fabrication. Disordered colloidal monolayers can be assembled through evaporatively driven convective assembly, a bottom-up process with a wide range of parameters impacting particle placement. Motivated by the photonic applications of such monolayers, in this review we discuss the quantification of monolayer disorder, and the assembly methods that have been used to produce them. We review the impact of particle and solvent properties, as well as the use of substrate patterning, to create the desired spatial distributions of particles.
\end{abstract}

Received 26th November 2021 Accepted 12th January 2022 DOI: $10.1039 / \mathrm{d} 1 \mathrm{nr} 07814 \mathrm{c}$ rsc.li/nanoscale

\section{Introduction}

The study of light interaction with disordered media plays a major role in many fields, from optical bioimaging, ${ }^{1}$ to atmospheric optics, ${ }^{2}$ to photonic material design. ${ }^{3}$ Light propagating in a disordered medium is diffused and attenuated by multiple scattering and absorption. ${ }^{4}$ While many studies have considered the analysis of light transport in common materials (powders, foams, biological tissues, etc.), the development of

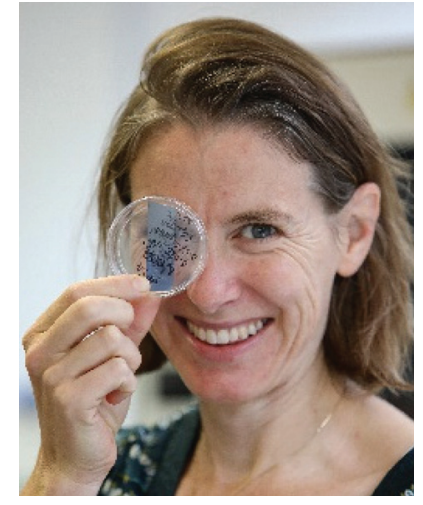

Glenna L. Drisko
Glenna L. Drisko is a CNRS researcher at the Institut de Chimie de la Matière Condensée de Bordeaux (ICMCB), with core interests in (nano)particle synthesis and assembly into $2 D$ and $3 D$ materials. She particularly focuses on high refractive index particles and their interaction with light. Her recent European Research Council funding 'Scatter' aims to synthesize silicon particles of complex shape and composition for extrainstitute in Paris. He entered the CNRS at the Laboratory for Photonics, Numerics and Nanosciences (LP2N) near Bordeaux. He is a recipient of the CNRS Bronze Medal. Currently, with his colleagues, he investigates how disordered nanostructures can be exploited for visual appearance design. 
top-down and bottom-up nanofabrication techniques in the past decades stimulated the emergence of a new research topic aiming to understand how disorder could be engineered to control the propagation, ${ }^{5,6}$ spatial confinement, ${ }^{7}$ and emission $^{8,9}$ of light. Research on disorder engineering in optics is in full swing. ${ }^{10-12}$

Disorder engineering seeks to exploit the fact that light scattered by random particle assemblies is affected by both the nature of the particles and their spatial arrangement. ${ }^{12}$ This is equivalent to the principle behind small-angle scattering techniques, ${ }^{13}$ where statistical information on the morphology of complex media is retrieved from scattering measurements. In translucent colloidal solutions, for instance, the scattering arises from both that of individual particles and the far-field interferences created by spatially correlated pairs of particles (here $\overrightarrow{\boldsymbol{q}}$ is the scattering wave vector with amplitude $q=|\overrightarrow{\boldsymbol{q}}|=\frac{4 \pi}{\lambda} \sin \frac{\theta_{\mathrm{s}}}{2}, \lambda$ is the light wavelength and $\theta_{\mathrm{s}}$ the scattering angle). The intensity of structural correlations can be expressed (to the lowest approximation) as $I(\overrightarrow{\boldsymbol{q}})=N P(\overrightarrow{\boldsymbol{q}}) S(\overrightarrow{\boldsymbol{q}})$ where $N$ is the number of particles in the cluster. The form factor $P$ and static structure factor $S$ describe respectively the scattering properties of the particles alone and the effect of their spatial arrangement on the scattered intensity. In addition to the influences of the size, shape and composition of the individual nano-objects, structural correlations in disordered systems constitute a valuable degree of freedom to spectrally modulate the amplitude and reshape the angular distribution of the scattered light. When passing from an ordered system to a disordered system, light transmission varies from collective light propagation (diffraction resonances) to individual particle scattering (diffusion). Tuning the particle-particle distance via order determines the degree of collective versus diffusive light propagation. Fig. 1 demonstrates how disorder effects light transport, with a preferred transmission direction in an ordered system and diffuse transmission in a disordered film. ${ }^{14}$ Ballistic light transport can thus be controlled via tuning the correlation distances.
In bulk three-dimensional (3D) correlated disordered materials, this modified light scattering translates into spectral variations of the diffuse reflectance and transmittance, yielding structural colors, ${ }^{15}$ and also engendering wave interference phenomena. These effects are of primary interest to the mesoscopic physics community. ${ }^{16}$ However, the realization of thick materials with controlled disorder remains particularly challenging. ${ }^{12}$

In random nanostructured surfaces, the modified light scattering is directly observed in the angular distribution of the reflected or transmitted light, which may also vary with the wavelength. ${ }^{17}$ Nanostructured surfaces are more accessible experimentally than their 3D counterpart and already find use in many applications, including photovoltaics, ${ }^{18-20}$ light emitting devices, ${ }^{21}$ and chemical and biosensing. ${ }^{22}$ Two-dimensional (2D) correlated disorder is ubiquitous in the living world, where nanostructured surfaces serve as anti-reflection coatings, ${ }^{23}$ and in thin film technologies, where metal-dielectric composites can exhibit a rich variety of optical properties, such as electromagnetic 'hot spots'. ${ }^{24,25}$ More recent years have witnessed a growing interest in disordered planar assemblies of resonant nano-objects, ${ }^{26-28}$ also known as "random metasurfaces". There is a collective effort to understand how the interplay between order and disorder leads to new or improved optical functionalities. ${ }^{14,21,29-37}$

These applications require particles with well-defined characteristics that can be assembled into large area surfaces. Advances in colloidal chemistry in the past decades have enabled the synthesis of a wide range of nano-objects with various shapes, sizes and compositions, and that can strongly interact with light, despite their small size. ${ }^{38-42}$ Bottom-up approaches generally do not give as much control over the shape and spatial arrangement of nano-objects as does topdown techniques, but they remain unrivalled in terms of scalability. Colloidal techniques are likely to play a major role in the future development of surfaces with controlled disorder.

In this review, we will discuss how disorder can be controlled during evaporation-induced self-assembly. In section 2,
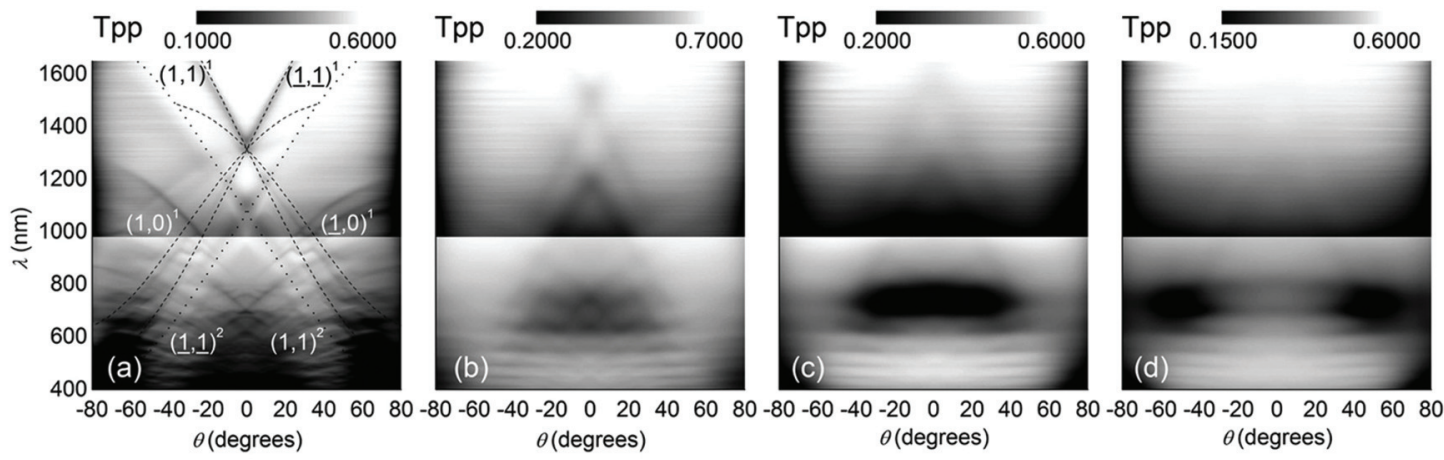

Fig. 1 Ballistic light transport decreases as disorder increases. Angle and frequency resolved transmission patterns are shown for films varying from (a) perfectly ordered to (d) slightly correlated particle-particle distances. The lines in (a) show the quasi-guided pathways. In this study, $1060 \mathrm{~nm}$ polystyrene microspheres were assembled with an increasing fraction of impurities introduced from a second population of $300 \mathrm{~nm}$ polystyrene particles. Figure reproduced with permission from ref. 14. Copyright 2016 Nature Publishing Group. 
we discuss controlled disorder in colloidal films, how it is defined and the sources of such disorder, with a discussion of the methods used to quantify it. Section 3 is devoted to the techniques used to self-assemble colloidal monolayers via evaporative processes. We describe the underlying physical principles which cause this behavior and the experimental approaches which use them. In section 4, we review examples of 2D colloidal assemblies with disorder induced through changing the size and shape of the assembled particles. We focus on the deliberate use of polydisperse particles to create disorder, and the impact of using non-spherical particles, such as cubes and rod-like particles to change assembly behaviors. In section 5 , we discuss changes in disorder achieved by modifying the dispersant properties through the inclusion of ions to screen particle-particle and particle-substrate interactions. Additionally, we look at how solvent evaporation rate through control of ambient pressure changes organization. Section 6 details the use of chemically and topographically patterned surfaces to control disorder. We first look at chemical patterning techniques to selectively deposit particles via patterned substrate functionalization with hydrophobic and hydrophilic functionality and the self-assembly of patterns using block copolymers. This is followed by a discussion of the use of topographically patterned substrates featuring traps or physical barriers to influence evaporatively driven particle assembly. Finally, we close with our conclusions and perspectives on these techniques and the future of this field.

\section{Order versus disorder}

\subsection{Defining controlled disorder}

In terms of particle assembly, the quintessential ordered system is that of a colloidal crystal, i.e., a regular structure of particles that extend over length scales much larger than themselves. Order is not necessarily synonymous with a periodic structure, regular crystal structures, with high degrees of positional order exist such as those of protein crystals, which possess random orientational disorder between individual proteins. Disorder is typically defined as deviation from an idealized ordered system. Within disordered structures, the position of the elements cannot be determined exactly and are instead described by statistical properties. Correlated disorder refers to systems in which variations in interparticle distance occur within a range. In the case of diffraction patterns from systems with correlated disorder, diffuse rings from continuous scattering rather than discrete points (from Bragg reflection) are observed. ${ }^{43}$

Disorder can be present in multiple ways. In the case of close-packed systems these include local defects (point/line defects and vacancies), particle polydispersity, poor particle packing, orientational disorder when using anisotropic particles, and inhomogeneous particle shapes or deposition patterns (Fig. 2). In the case of non-close packed systems, this also includes irregular spatial distributions of particles. In $2 \mathrm{D}$ particle assemblies, the level of disorder can be quantified by correlation functions, a pair correlation function for the position of the element, an angular correlation function for the orientation of the particles. However, some other types of disorder would not be apparent in these correlation functions, such as point lattice defects and polydispersity in non-close packed assemblies. These types of disorder can be seen by other quantification strategies, such as the bond orientational order parameter.

Truly disordered systems will have no crystalline domains. In this case, the pair distribution function is equal to one and no correlation is found at any length scale. ${ }^{44}$ Zero correlation is not truly achievable in particle assemblies because particles cannot penetrate each other and thus must exhibit some degree of correlation.

Controlled disorder refers to the engineering of systems to be randomly organized but restricted within a predetermined set of parameters. In the case of colloidal films, such systems will have a fixed level of correlation between individual particles in terms of their spatial and orientational organization. As of today, relatively few research groups have intentionally introduced disorder into two-dimensional assemblies of particles, ${ }^{12}$ and even less so in the case of films assembled through evaporative techniques. However, many of the systems currently found in the literature do offer routes to deliberatively introduce disorder. Hence, we discuss the conditions that have been observed experimentally to provide an increase in disorder in convectively assembled films.

\subsection{Quantifying disorder in 2D colloidal films}

Several techniques exist to quantify disorder in colloidal films including Fourier analysis of microscopy images, X-ray or neutron scattering, radial distribution functions, and Voronoi diagrams and Delaunay triangulations. We will briefly address each of these, and encourage the reader to consult Lotito and Zambelli's recent comprehensive reviews on this topic for further insights. ${ }^{45,46}$ Disorder can be measured on different scales both the long-range via macroscopic techniques, such as Fourier analysis and scattering techniques; and short-range through analysis of local bond order. We discuss these techniques in the following paragraphs.

A fast and convenient approach to quantifying order in colloidal films is Fourier analysis of images. Fast Fourier transform algorithms allow images of particle monolayers to be analyzed in terms of their component spatial frequencies with minimal processing time. Highly ordered monocrystalline films produce ordered patterns of high intensity spots, whereas these spots smear into circular rings for polycrystalline samples. As disorder is increased in the sample, these features drop in intensity and eventually devolve into a $2 \mathrm{D}$ Gaussian. The radially averaged Fourier transform intensity can reveal the length scales of ordered regions, examples of this sort of analysis are ubiquitous in literature. ${ }^{14,47,48}$ The quality of the results from this technique is highly dependent on parameters such as image contrast, resolution and focusing because they are calculated from pixel intensities, rather than particle positions. 

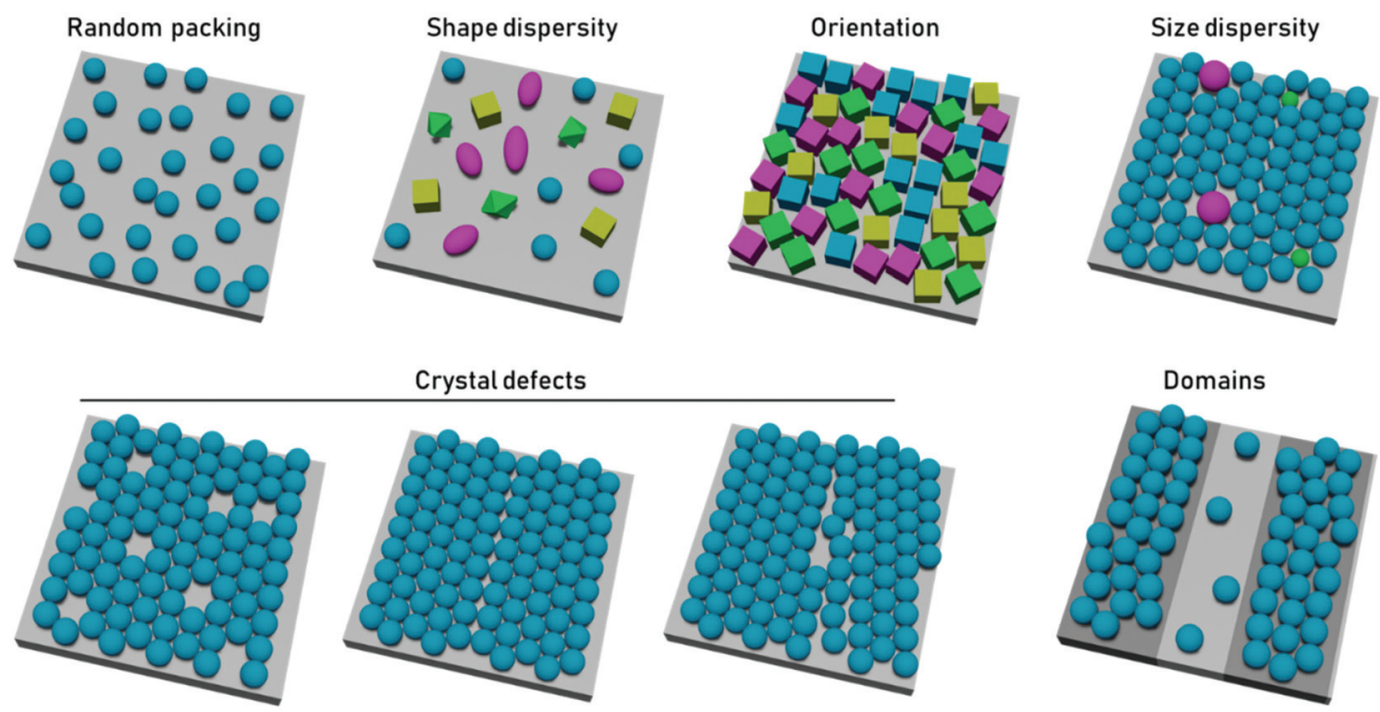

Fig. 2 The most common sources of disorder are from random, non-close packing, from dispersity in particle shape, orientation, or size, from crystal defects, such as point or line defects and grain boundaries, and from particle placement outside desired domains.

Crystalline order can be directly probed through scattering techniques which analyze a large area of the monolayer, providing information about disorder at the macroscopic level. While light diffraction ${ }^{14,49-54}$ and neutron scattering ${ }^{55,56}$ have been used to characterize colloidal films, small-angle X-ray scattering (SAXS) is a far more common method to characterize disorder in such films. ${ }^{57-66}$ Light diffraction is limited to films containing particles with diameters above the optical diffraction limit, whereas SAXS and neutron scattering can resolve much smaller structures. As with Fourier analysis, the formation of peaks and rings is indicative of crystalline structure. Disordered samples present Gaussian scattering patterns. ${ }^{53,61}$ The intensity and width of the peaks in the scattering patterns of monolayer films provide information about the level of order within a film and can be analyzed to provide a global value for the layer in the form of a structure factor. The sine Fourier transform of these patterns is the pair correlation (or radial distribution) function, $g(\vec{r})$, which expresses the probability of finding a particle at a given radial distance from another particle. ${ }^{67}$

Low level image analysis techniques analyze the structure of the film in terms of particle positions. These are identified from images of particle films obtained via atomic force microscopy (AFM), electron microscopy, or optical microscopy. The particle positions can be analyzed statistically, providing a quantitative evaluation of the spatial variation of monolayer structure. The pair correlation function can be calculated directly from this dataset. ${ }^{68-70}$ However, more complex analyses can provide further insight into local order variation throughout the sample. The simplest of these is VoronoiDelaunay analysis, where the Voronoi cell of each particle is determined. The edges of each cell are defined as being equidistant between two nearest neighbors and the vertices equidistant between three nearest neighbors (Fig. 3). Each edge is thus intersected by a single nearest neighbor bond. For a (a)

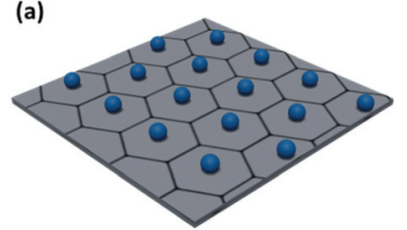

(b)

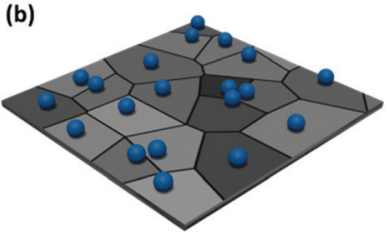

Fig. 3 Example Voronoi diagrams for (a) a highly ordered non-close packed monolayer and (b) a disordered non-close packed monolayer.

perfect hexagonally close packed crystal, the number of nearest neighbors, $N_{j}$, is six. More disordered lattices will feature Voronoi cells of varying area ${ }^{71,72}$ and differing number of sides. ${ }^{47,73,74}$ Statistical analysis of the population of these cells enables the calculation of values describing the entire population, such as the variance or global Voronoi entropy, $S_{\mathrm{vor}}{ }^{47,75}$

Another common approach is the quantification of local bond order, though the analysis of changes in the angles between nearest neighbors, which can be expressed through the bond-orientational order parameter, $\psi_{n}:{ }^{76-79}$

$$
\psi_{n}\left(\overrightarrow{\boldsymbol{r}}_{j}\right)=\frac{1}{N_{j}} \sum_{j}^{N_{j}} \exp \left\{i n \theta_{j k}\left(\overrightarrow{\boldsymbol{r}}_{j}\right)\right\}
$$

where $n$ is the symmetry order $(n=6$ for hexagonally closepacked, $n=4$ for square), $\theta_{j k}\left(\vec{r}_{j k}\right)$ is the angle between a reference direction and the vector between particle centers $j$ and $k$. Given a sufficient definition of 'nearest neighbor', $\psi_{6}$ can be used as a measure of the local bond order throughout a $2 \mathrm{D}$ crystalline lattice. ${ }^{80}$ For well-ordered 2D hexagonally closepacked lattices, $\psi_{6}$ will vary between 0.9 and $1 .^{81}$ It is widely used as a measure of the order in $2 \mathrm{D}$ particle assemblies (i.e. ref. 72, 74, 78 and 81-89). Additionally, the complex phase of 
this function, $\theta_{n}=\frac{1}{6} \arg \left(\psi_{n}\right)$, can be used as a direct measure of local crystalline ordering. $\theta_{6}$ varies between $0^{\circ}$ and $60^{\circ}$ for a hexagonally close-packed crystal. Any variation in the phase or amplitude of this parameter is indicative of disorder in the lattice due to polycrystallinity or the inclusion of lattice defects.

There are limitations to this approach however. The results produced by this analysis are highly sensitive to the definition of nearest neighbor used. This is normally defined as any particle center inside a radius of some multiple of the particle diameter, $D$, for close-packed monolayers this is typically between $\sim 1.2 D$ and $\sim 1.4 D$ as determined by the position of the first trough in the radial distribution function, $g(\overrightarrow{\boldsymbol{r}}) .{ }^{90}$ However, for non-close packed monolayers, such as that depicted in Fig. 3b, these definitions become problematic due to highly variable bond distances resulting from low packing fractions and the presence of voids. It is also not continuous as a function of particle coordinates, making it less practical as a mathematical parameter. $^{91}$

To solve these problems, $\psi_{n}$ can be weighted to reflect local geometric features in the lattice by combining this protocol with Voronoi analysis. In this case, a relative length factor, $l_{j k}(\overrightarrow{\boldsymbol{r}}) / L$, is included in the definition of $\psi_{6}$, where $l_{j k}(\overrightarrow{\boldsymbol{r}})$ is the length of the intersected Voronoi edge and $L$ is the perimeter of the Voronoi cell $\left(=\sum_{k}^{N} l_{j k}(\overrightarrow{\boldsymbol{r}})\right):{ }^{90,92-94}$

$$
\psi_{n}^{(\mathrm{msm})}\left(\overrightarrow{\boldsymbol{r}}_{j}\right)=\sum_{j}^{N_{j}} \frac{l_{j k}}{L} \exp \left\{\operatorname{in} \theta_{j k}\left(\overrightarrow{\boldsymbol{r}}_{j}\right)\right\} .
$$

This parameter robustly describes the order within a $2 \mathrm{D}$ colloidal crystal. For a perfect hexagonally close packed crystal, this parameter returns to the form given in eqn (1). This formalism is functionally identical to the Minkowski structure metric used elsewhere. ${ }^{47,91,93}$ Images of assembled particles can be analyzed to find regions that demonstrate predominantly 4-, 5-, or 6-fold symmetry, distinguishing between regions of hexagonally close packed crystallinity and defects. ${ }^{95}$ The local crystalline phase, $\theta_{n}$, can also be analyzed to identify grains. ${ }^{81}$

There are also techniques to characterize the component crystallinities within a monolayer to give a more holistic description. One such example is persistent homology ${ }^{96-100}$ which describes the particles in terms of topological parameters such as the Betti numbers. Other statistical techniques, such as principal component analysis, can also identify small changes in global order between different films. ${ }^{100}$

\section{Fundamental concepts of evaporative particle self-assembly}

While several approaches exist for the self-assembly of colloidal films, we focus here purely on the use of evaporative self-assembly. Evaporative assembly techniques offer a simple, scalable, and cost-effective route to prepare colloidal films, which make them attractive for future industrial processes. Here, we discuss the underlying principles behind these techniques, and the available protocols which use them in the preparation of colloidal films.

In evaporatively driven particle assembly, a volume of colloidal solution is directed over a substrate establishing a triplephase contact line between air, the solvent, and the substrate. Good wettability ensures the formation of a continuous film. Without this, dewetting can occur and islands can form. This can be particularly problematic if the contact angle is too high $\left(>20^{\circ}\right)$, because no particles will be deposited (Fig. 4). But under ideal conditions, tightly packed and homogeneous layers of particles will result. Depending on the technique employed, the solvent recedes due to either substrate withdrawal from the solution or from solvent evaporation. Subject to the rate of solvent recession, there are two regimes known as which are referred to as the 'convective' regime and the 'Landau-Levich' regime.

Regime I: convective assembly: Also known as the 'capillary' regime, convective assembly is characterized by particles being brought to the drying line by an evaporatively-driven flow. It occurs at low speeds $\left(\lesssim 0.1 \mathrm{~mm} \mathrm{~s}^{-1}\right.$ in ethanol at $\left.30{ }^{\circ} \mathrm{C}\right)$, where evaporation in the meniscus is the dominant process driving particle transport. Particles concentrate close to the drying line and become trapped at the meniscus edge, forming dense particle arrangements on the substrate. The particles within the forming film are then bound by interparticle and particle-substrate forces. Further evaporation then produces a dry film. Convective particle flow is dependent on solvent evaporation rate, the buoyancy of the particles, the force of Brownian motion on the particles, and the substrate withdrawal rate. Evaporative flux can be controlled through ambient temperature, pressure, and humidity, alongside solvent volatility.

However, working against convective particle transport is an osmotic pressure, expressed by Fick's law: a higher particle concentration in the drying line leads to diffusive flow towards

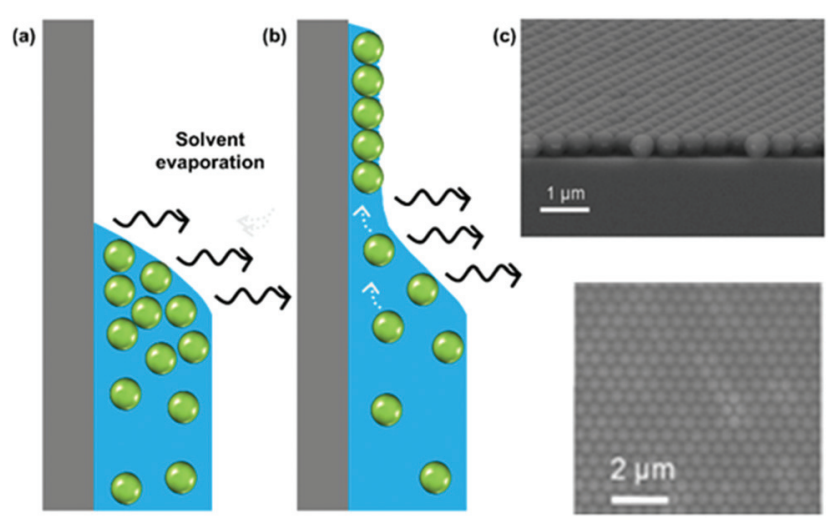

Fig. 4 (a) A high contact angle, where particles are not pinned to the surface and no film deposition is achieved. (b) A good wetting with a contact angle of less than $20^{\circ}$, where convective flux brings particles to the drying line. (c) A continuous monolayer of polystyrene latexes. (c) Reproduced with permission from ref. 101. Copyright 2007 American Chemical Society. 
the bulk solution. ${ }^{103}$ Particle size, shape, and density, as well as interparticle interactions, contact angle, and solvent volatility, all influence whether convection or diffusion is dominant. Collective particle transport towards the triple line is faster for neutrally charged particles than for positively or negatively charged particles. ${ }^{103}$ This is due to increased diffusion rate in concentrated dispersions of charged particles. For charged particles, interparticle interactions are more important than substrate-particle interactions in determining film thickness. ${ }^{103}$

The number of particle layers, $k$, in the obtained films is inversely proportional to the substrate velocity speed. ${ }^{104-106}$ In this regime, continuous film formation can only be achieved if the substrate velocity, $v_{\mathrm{s}}$, is equal to the crystallization velocity, $v_{\mathrm{c}}$, as expressed by the Dimitrov-Nagayama equation: ${ }^{104}$

$$
v_{\mathrm{s}}=v_{\mathrm{c}}=\frac{\beta l}{0.605} \frac{j_{\mathrm{e}} \phi}{k D(1-\phi)}
$$

where $\beta$ is an experimentally determined constant representing particle-particle and particle-substrate interactions, $j_{\mathrm{e}}$ is the evaporative flux, $\phi$ is the particle volume fraction, and $D$ is the particle diameter.

As substrate velocity is decreased, the well-known phenomenon of 'stick-slip' becomes increasingly pronounced (Fig. 5). This is caused by contact-line pinning, where the meniscus becomes stuck and then elongated by the motion of the sub- strate. Eventually the surface energy of the meniscus becomes too great, leading to the contact line suddenly 'slipping' to the next pinning line. ${ }^{107,108}$ This process is periodic and causes particle deposition to occur in regular bands parallel to the meniscus.

Regime II: 'Landau-Levich' assembly: Also known as the 'advective', 'draining', or 'viscous drag' regime, Landau-Levich assembly occurs at high speeds $\left(\gtrsim 1 \mathrm{~mm} \mathrm{~s}^{-1}\right.$ in ethanol at $25^{\circ} \mathrm{C}$ ). Here viscous forces within the liquid dominate. In this regime, a static meniscus at the evaporative drying front no longer exists and instead the solvent viscosity produces a thin continuous film of the coating solution having approximately constant thickness across the substrate. This film then dries through draining leading to the deposition of particles on the substrate. The shearing induced by draining can lead to vertically striped deposition patterns under some conditions. ${ }^{102}$ In the formation of Landau-Levich films, the viscous, gravitational, and surface tension forces in the trapped fluid layer exist in equilibrium. The Landau-Levich equation can be used to describe the thickness of this fluid layer, $h_{0}$ :

$$
h_{0}=\frac{\left(\eta v_{\mathrm{s}}\right)^{\frac{2}{3}}}{\gamma^{\frac{1}{6}}(\rho g)^{\frac{1}{2}}}
$$

where $\gamma$ is the air-liquid surface tension, and $\rho$ is the density of the solvent. Hence, the thickness is proportional to the $\frac{2}{3}$ (a)

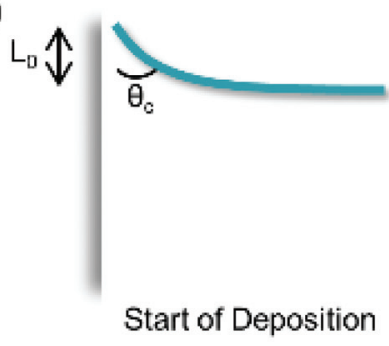

Start of Deposition
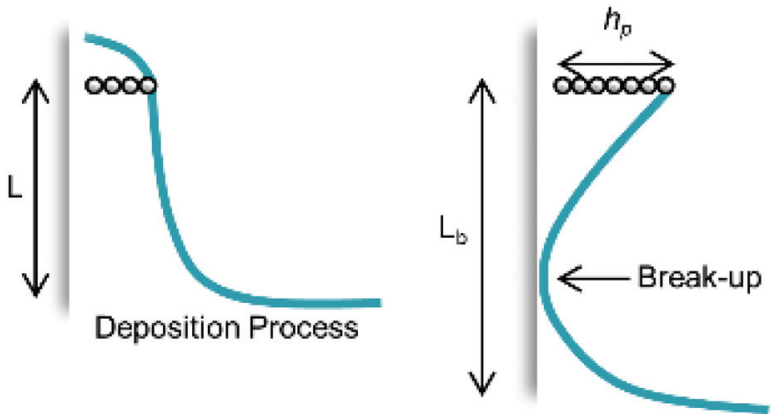

Meniscus Touch-down
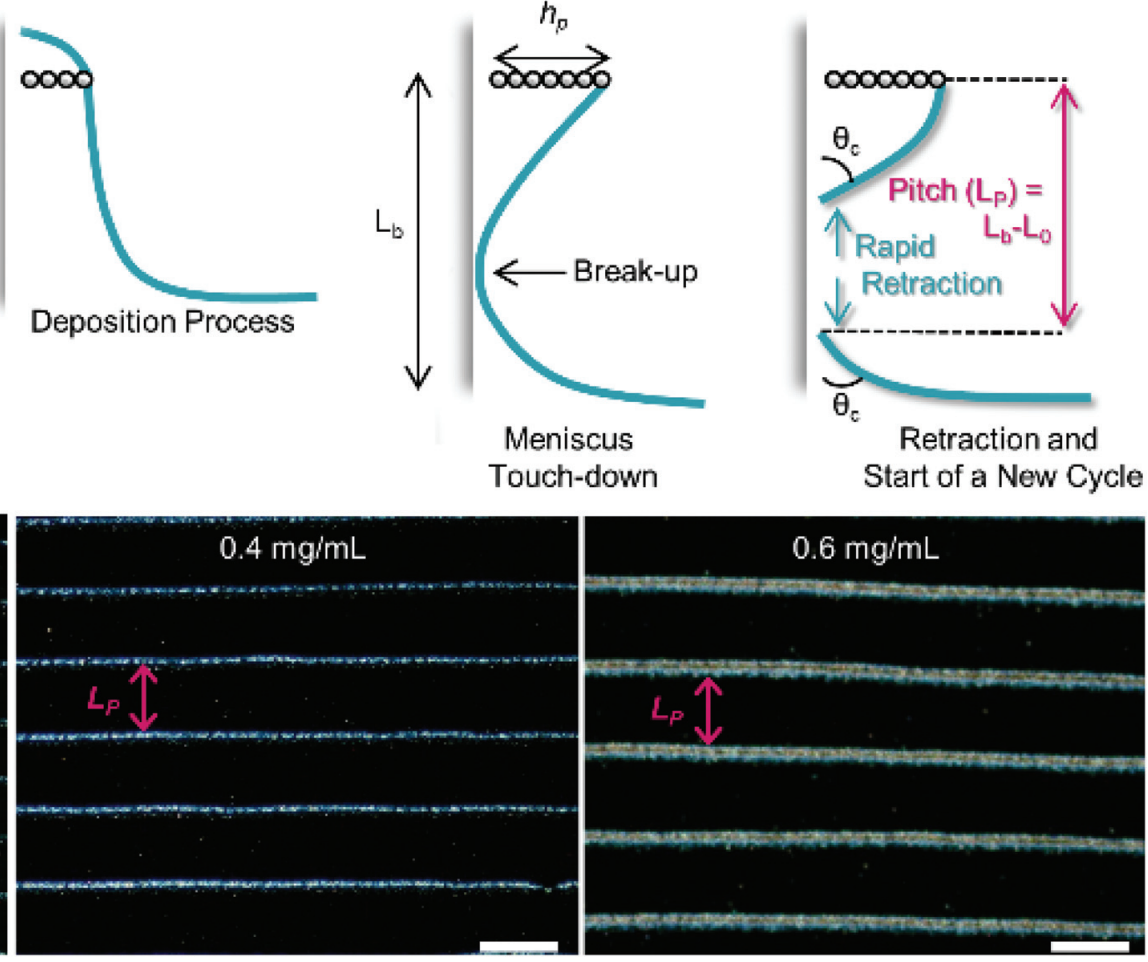

Fig. 5 (a) The process of stick-slip, where particles pin the meniscus, the meniscus becomes stretched, the meniscus touches down, breaking contact with the bulk solution and a new deposition begins. (b) Optical microscopy images showing the dependence of pitch on particle concentration in the solution (concentrations of $0.2,0.4$ and $0.6 \mathrm{mg} \mathrm{mL}^{-1}$ from left to right). The scale bars represent $100 \mu \mathrm{m}$. Reproduced with permission from ref. 102. Copyright 2020 American Chemical Society. 
power of the substrate velocity and solvent viscosity, and is inversely proportional to the $\frac{1}{6}$ and $\frac{1}{2}$ powers of the air-solvent surface tension and solvent density respectively. ${ }^{109,110}$ The effects of convective transport from the bulk solution are negligible in this regime and formation occurs entirely through the pulling of the liquid film. Palma and Lhuissier confirmed experimentally that the number of particle layers in the dry film also follows the same power relationships with an additional dependence on volume fraction of the particles within the liquid film. ${ }^{111}$ Hence the solvent viscosity, surface tension, and density serve as important parameters in controlling film deposition in this regime alongside the substrate velocity.

Transitional regime: Between the convective and LandauLevich regimes, there exists a transitional regime where both assembly mechanisms occur in tandem. It is here that the thinnest possible films are achieved (withdrawal rate between 0.1 and $1 \mathrm{~mm} \mathrm{~s}^{-1}$ in ethanol at $\left.25{ }^{\circ} \mathrm{C}\right) .{ }^{112}$ In this regime, a liquid film is formed, but evaporation occurs faster than the liquid film can drain. A drying front is established at the top of the liquid film causing convective assembly to occur within the liquid film itself. ${ }^{113}$

In all regimes, the ability to form good quality films requires the prevention of dewetting by using substrates with good wettability. Both assembly processes are sensitive to changes in surface tension, which can be controlled through solvent selection and the inclusion of surfactants in the coating suspension. ${ }^{48,104,114}$

Colloidal stability, through either electrostatic or steric approaches, is crucial to avoiding sedimentation during the coating process. ${ }^{115}$ Sedimentation is particularly prevalent for larger particles, where surface functionalization can no longer overcome sedimentation forces on the particle. Remedial actions to address this problem for larger particles include: increasing the rate of evaporative flux (increasing deposition speed), using solvents with higher viscosity (reduced sedimentation velocity) or density (increased particle buoyancy). ${ }^{113}$

There are several ways to deposit films using these principles, the most common being: drop-casting, dip-coating, and drag-coating. We point interested readers to a recent in-depth review which discusses this topic for further insights on the assembly of these films. ${ }^{45}$

\subsection{Drop-casting}

The simplest method of evaporative assembly is that of droplet evaporation or drop-casting, ${ }^{116-121}$ which is the free evaporation of a droplet of colloidal solution placed directly on a substrate. The area of the substrate that can be coated is thus restricted to the size of the droplet. As the suspension evaporates, particles are drawn towards the contact line resulting in the 'coffee-ring' pattern, in which thick particle deposits occur at the edges of the droplet. ${ }^{122}$ Several adjustments have been proposed to avoid coffee-rings, including introducing a cell wall, controlling $\mathrm{pH}$, and drying in a solvent-saturated atmosphere. ${ }^{123-126}$ It is difficult to control such processes, and inhomogeneous deposition is still an issue, with coatings often simultaneously containing regions of multilayer, monolayer and submonolayer.

\subsection{Dip-coating}

This technique has been used to coat substrates with polymers, ${ }^{105,127-129}$ biomolecules, ${ }^{130-134}$ and, most relevantly, colloidal solutions. ${ }^{104,135,136}$ Typically, dip-coating is performed by submerging a substrate in a reservoir containing a coating suspension and then withdrawing it vertically at a known velocity. Many factors impact the physical qualities of the film: the withdrawal rate, the number of dip cycles, the contact angle (substrate nature and solvent polarity), the evaporation rate (e.g., chamber temperature and pressure, solvent volatility, and relative humidity) and the solution properties (e.g., viscosity, surface tension, and the particle concentration), as well as the particle properties (surface chemistry, size, shape, and material).

Many reports in the literature control ambient humidity, ${ }^{137,138}$ pressure, ${ }^{115}$ or introduce airflow over the meniscus ${ }^{139,140}$ to regulate the evaporation rate of the coating solution. Control of the formed film can also be exerted through modification of interparticle interactions through changing colloidal surface charge via the solvent $\mathrm{pH}^{141}$ or screening these interactions through the inclusion of electrolytes $^{114}$ or charged polymers ${ }^{135}$ in the coating solution.

Compared to other techniques such as spin coating, relatively little coating solution is wasted, as the material left in the reservoir can be reclaimed. However, there are losses to the walls of the reservoir and the other substrate surfaces. The area that can be coated is in theory only limited by the sizes of the substrate and the reservoir. Also, the large volume of supension required to fill the reservoir (relative to the amount used in coating the substrate) means that this technique is not ideal for small-batch processes or suspensions that can only be prepared in a small volume. It is a relatively simple experimental set-up, although requires good control of many parameters to yield reproducible films.

\subsection{Drag-coating}

A closely related technique to dip-coating is drag-coating, or 'doctor-blade' coating, which is the horizontal counterpart to dip-coating. It similarly has found a use for coating substrates with biomolecules, ${ }^{142-145}$ polymers ${ }^{146-148}$ and particles. ${ }^{149-151}$ In this set-up, a small volume of the coating solution placed between the substrate and a blade is guided across the substrate. Capilary forces hold the colloidal solution in contact with both the substrate and blade, and assembly occurs at the trailing edge of the volume.

In the angled-blade configuration, the blade can be moved in either direction with respect to the substrate. When moved in the forward direction, assembly occurs below the blade, ${ }^{87}$ and when moved in the reverse direction assembly occurs at the contact line which trails the blade. ${ }^{152,153}$ Higher crystallization velocities are observed in the reverse direction. ${ }^{152}$ Dragcoating involves the same parameters as dip-coating, but also includes additional considerations about properties of the 
blade, such as the angle, ${ }^{87,151,154}$ surface functionalization, ${ }^{87}$ and height above the substrate. ${ }^{155}$ The blade applies pressure to the meniscus and through variation of the blade height, the curvature of the meniscus can be reduced, in turn minimizing contact line pinning and variation in the film thickness. ${ }^{155}$

Drag-coating apparatuses are typically more compact than dip-coaters but are limited by the small volume of coating solution used, which restricts the area that can be coated. There have been some attempts to modify this technique to enable the solution to be continuously fed below the blade using a syringe pump at a rate matched to the evaporative flux, thus enabling continuous coating over large areas. ${ }^{156}$ The relative fluxes of both the solvent and particles must be matched to maintain a constant concentration in the reservoir and make the process truly continuous.

Several changes to this technique have been implemented, such as modifying interparticle interactions through $\mathrm{pH}$ control, ${ }^{143}$ or electrostatic screening using electrolytes, ${ }^{157}$ as well as changing the evaporation rate. ${ }^{145,153}$ Some approaches assist monolayer formation through the vibration of the sample in the plane of meniscus travel (lateral vibration). Vibrational frequencies in the range 20 to $50 \mathrm{~Hz}$ show a significant increase in the range of blade velocities that produce continuous monolayers. ${ }^{158-160}$ Vibrations out of the plane of the substrate (vertical vibration) show similar results at higher frequencies $(3 \mathrm{kHz}){ }^{161}$

In summary, two regimes of particle assembly are accessible by these techniques. In the Landau-Levich regime there is little that can be done to influence the degree of disorder; only the particle density can be regulated. By contrast, the convective assembly regime has a wide range of parameters with which disorder can controlled. These will be explained more fully in the next few sections.

\section{Using particle properties to control disorder}

One strategy to control particle deposition is modification of the particles' physical characteristics. In this section we will review the effects of changing particle polydispersity and the use of anisotropically shaped particles on their assembly.

\subsection{Disorder arising from particle size polydispersity}

Typically, polydispersities below $10 \%$ have little impact on the crystalline structure. ${ }^{162}$ Polydispersity being defined as $\delta=\sqrt{\left(\left\langle D_{i}^{2}\right\rangle-\left\langle D_{i}\right\rangle^{2}\right) /\left\langle D_{i}\right\rangle}$, where $D_{i}$ is the particle diameter and angular brackets indicate the ensemble average. ${ }^{162}$ Polydispersities between 10 and $15 \%$ can lead to partially ordered systems, with values $>15 \%$ producing disordered systems. It is energetically preferable for hard spheres to be packed in crystalline domains compared with disordered arrays, as the thermodynamic stability increases upon crystallization. It has been observed that due to chemical interactions between spheres, polydisperse samples will segregate into mul- tiple crystals with different unit cell sizes. ${ }^{163}$ Alternatively, an $A_{x} B_{y}$ unit cell, where $A$ and $B$ are spheres of different diameter, is also an energetically favorable arrangement. Thus, disorder should be induced via kinetic quenching or by using chemical forces or physical topography.

Lau and Russel have described the effect of surface charge on the organization of size polydisperse particles by dragcoating (Fig. 6). ${ }^{164}$ Lateral capillary thickness, $h$, of the liquid film is greater than or equal to the particle diameter, $D$. Once the liquid film thickness drops below the particle diameter, capillary forces pull the particles together. The smallest particles are still free to move, while the larger particles are immobilized. However, when the suspension is deionized, lateral movement is not determined solely by capillary pressure. It seems that the uniformity of the meniscus curvature is lost when the solution is deionized, leading to inhomogeneous capillary forces and a loss of order. Moreover, the repulsive double layer around the particle, rather than the core diameter, determines the effective particle size and thus their movement. Smaller particles have effectively the same lateral mobility as the larger particles and are incorporated into the assembly, rather than segregating to the edge of the ordered domains formed from larger particles. In summary, to control disorder, capillary forces increase order when the electrostatic interactions are higher.

Ions in the solvent create more homogeneous capillary forces, particularly when using hydrophobic particles, such as polystyrene. Thus, by tuning the electrostatic properties, the degree of disorder can be tuned in a polydisperse system.

The impact of polydispersity is shown by assembling monolayers of both monodisperse and polydisperse particle suspensions. ${ }^{165}$ The polydisperse sample led to amorphous films with a volume filling fraction between 42 and 54\%, compared with between 57 and $61 \%$ for monodisperse suspensions. In the crystalline monolayers, brilliant angle-dependent colors

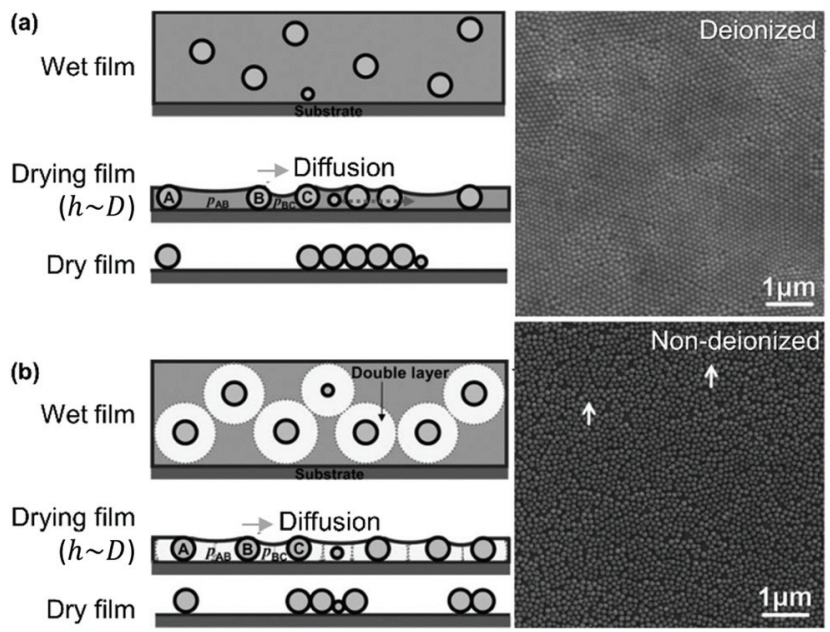

Fig. 6 Particle monolayers prepared using silica particles in (a) non-deionized and (b) deionized water show differing degrees of order as a function of polydispersity. Figure adapted with permission from ref. 164. Copyright 2014 John Wiley \& Sons. 
could be observed, due to scattering from close-packed domains of varying orientation within the film. This effect was absent in the disordered monolayer, where if color is observed, it is from interferences within the film rather than crystalline domains.

\subsection{Controlling disorder with particle shape}

Introducing anisotropy into convectively assembled systems has important implications because it changes both interparticle capillary interactions and restricts the possible packing configurations. It also introduces a new form of disorder in the form of angular correlation, which is unquantifiable when using isotropic particles. We focus here on films using rod-like and cubic particles.

4.2.1. Rods and wires. Anisotropic nanomaterials do not travel in the same way as elongated macroscopic objects in a current (e.g., a stick in a stream). Instead they tumble in a Jeffrey orbit, meaning that they precess as they as travel through the solution. ${ }^{166}$ As the film dries its thickness will become thinner than a Jeffrey orbit, at which point the particles will be dragged along the surface. Thus, lubrication of their displacement (e.g. using polymers, very small nanoparticles, or electrostatic repulsion from the substrate), will increase the degree of order in their packing. ${ }^{102,167}$

We used convective assembly to control the disorder in bands of tightly packed silica helices having a wire-like structure. ${ }^{102}$ Ordered assembly was possible in both the LandauLevich and convective assembly regimes (Fig. 7). At low speeds, in the convective regime, the helices oriented themselves parallel to the meniscus and a high degree of order was obtained, classified by order parameter $S$. Defined as $S=$ $\left\langle 2 \cos ^{2} \theta-1\right\rangle$ where $\theta$ is the angle between helices and angular brackets indicate the ensemble average. When $S=1$, the nanoobjects have no orientational disorder, and when $S=0$ the nano-objects have a random angular distribution. In the convective assembly regime, the quantity of polymer additive, that is polyacrylic acid, determined the degree of order. In absence of polymer, the assembly was completely random. Silica: polymer mass ratios of $1: 5$ to $1: 20$ were assembled, producing order parameters between 0.74 and 0.86 . The order parameter was increased further, attaining $S=0.95$, by controlling the concentration of helices in solution. Thus, by tuning the quantity of nano-objects and the amount of lubricant in the drying line, the degree of disorder can be tailored.

The aspect ratio in assembled nanorods has a significant impact on the degree of order. ${ }^{168-170}$ In the case of rigid nanoobjects, small aspect ratios provide more disordered films, with higher alignment for larger aspect ratios. ${ }^{170}$ With more flexible nanowires, a longer aspect ratio led to a higher degree of disorder, ${ }^{171}$ unless a significant shear force was applied. ${ }^{172}$ For these objects, a balance is struck between Brownian and shear forces. ${ }^{172}$

As in the case of spherical particles, the use of particles with a strong surface charge result in a higher degree of organization. ${ }^{170}$ Moreover, a faster rate of convective flow increases the degree of order. ${ }^{169}$ However, electrostatic charge also plays a role in particle-particle interactions. In one study, in the
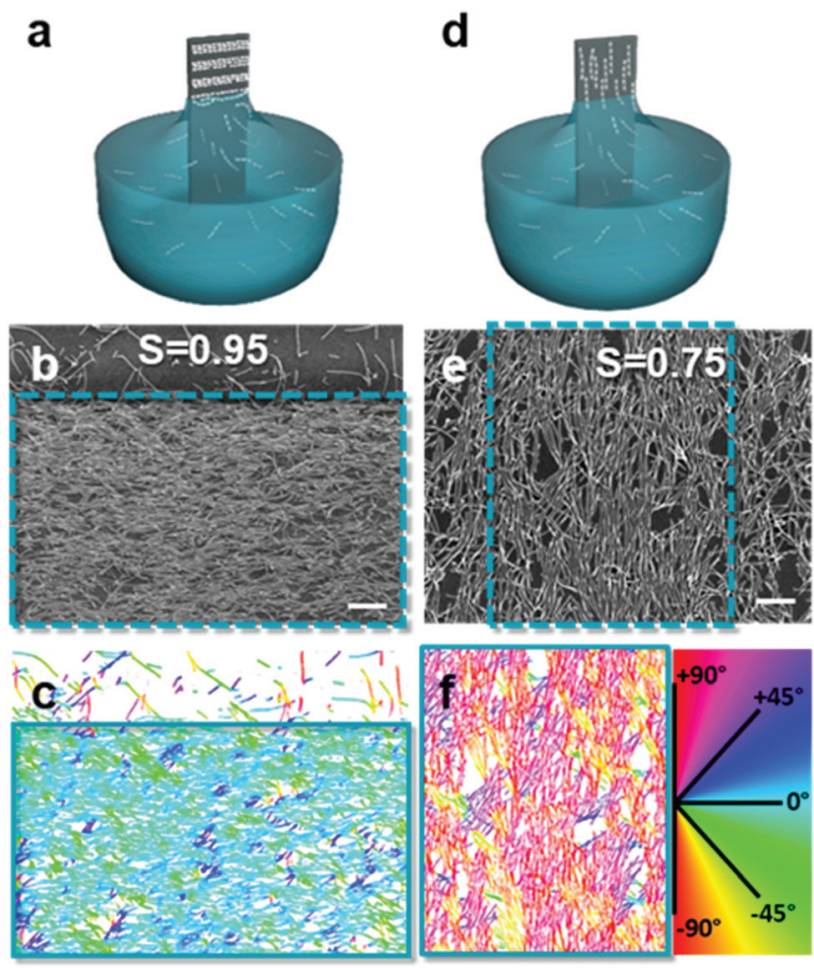

Fig. 7 Assembly in the (a) convective and (d) Landau-Levich regimes, where the ( $b$ and $e$ ) degree of disorder between assembled helices varies. (c and f) The order parameter, $S$, was obtained by treating the images to determine the orientation of the helices relative to the withdrawal direction. Scale bars represent $1 \mu \mathrm{m}$. Reproduced with permission from ref. 102. Copyright 2020 American Chemical Society.

absence of counter-ions, lateral repulsion between rod-like particles led them to predominantly assemble in a head-to-tail arrangement. ${ }^{173} \mathrm{~A}$ faster rate of convective flow has been found to increase the degree of order. ${ }^{169}$ Conversely, an evaporation rate that is too high has been found to position nanorods before they have the time to organize, creating more disordered materials. ${ }^{168}$

By using the lessons learned from spheres, the degree of order can be rather easily tuned in the assembly of anisotropic rods and wires. These objects tend to align either head-to-tail or side-to-side, depending on various interparticle forces. Aligned, non-close packed assemblies are not observed in absence of an external field or a template. More typically, during the evaporative assembly of these objects, highly aligned and tightly packed systems are observed, where higher aspect ratios increase the degree of order.

4.2.2. Cubes. During convective assembly, cubic particles orient themselves relative to their neighbors and irrespective of the growth direction of the film. ${ }^{174}$ Cubes assemble themselves into different types of packings, referred to as $\Lambda_{0}$-lattices, $\Lambda_{1}$-lattices, square lattices, and disordered assemblies (Fig. 8). ${ }^{95}$ The disorder observed in these assemblies is primarily due to rotational disorder and changes in lattice packing. Lattice structure was determined by comparing the distribution of angles between all of the nearest neighbors of each particle. ${ }^{95}$ 

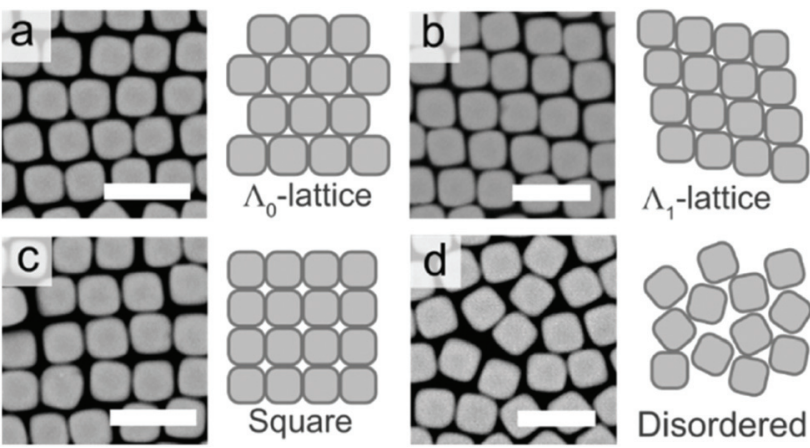

Fig. 8 The (a) $\Lambda_{0}$-lattice, (b) $\Lambda_{1}$-lattice (c) square lattice, and (d) disordered assembly patterns observed in SEM micrographs. Scale bars represent $2 \mu \mathrm{m}$. Reproduced with permission from ref. 95. Copyright 2019 American Chemical Society.

Meijer et al. examined the effect of corner sharpness on the preferred orientation and lattice of cube assemblies (Fig. 9). ${ }^{95}$ Corner sharpness was controlled by changing the thickness of a silica shell around a cubic hematite particle; thicker coatings produced more rounded nano-objects. Cubes were described as superballs satisfying the following relationship:

$$
\left|\frac{x}{a}\right|^{m}+\left|\frac{y}{a}\right|^{m}+\left|\frac{z}{a}\right|^{m} \leq 1
$$

with corner sharpness being parameterized by the shape parameter, $m,(m=2$ for a sphere, $m=\infty$ for a cube), and $a$ is half the edge length, $L$. Nanocubes with sharper corners produced assemblies with more regular orientation and a stronger pre-
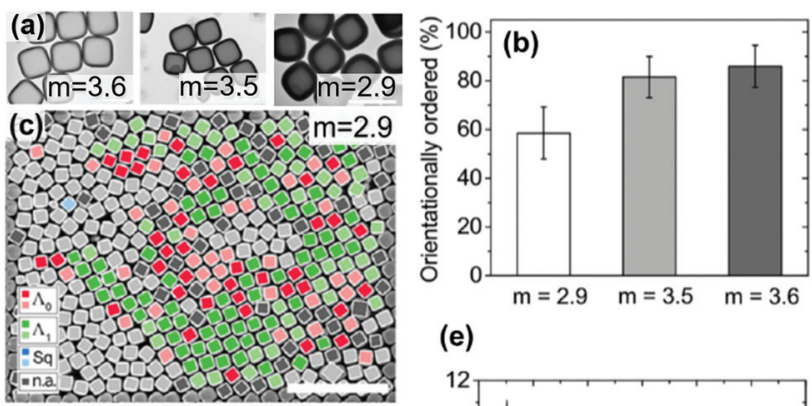

(e)
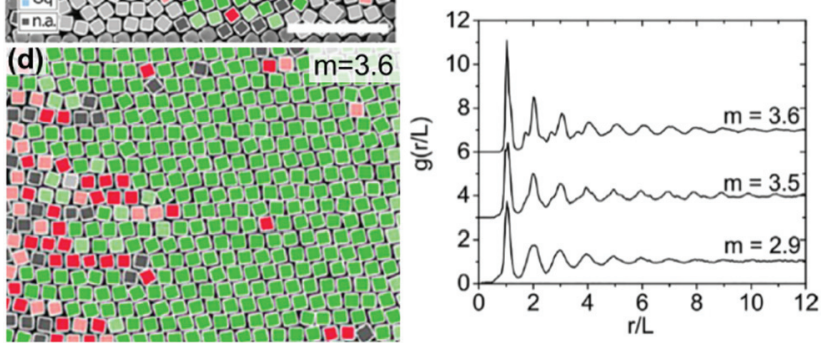

Fig. 9 (a) TEM images of cubes with different corner sharpness. The scale bars represent $1 \mu \mathrm{m}$. (b) The \% of cubes with orientational order for cubes with differing corner sharpness. SEM images of cubes (c) $m=$ 2.9 and (d) $m=3.6$ (defined in eqn (5)) with colors indicating the orientation and organization of cubes relative to neighboring cubes. The scale bars represent $5 \mu \mathrm{m}$. (e) An average radial distribution for cubes of differing corner sharpness. Reproduced with permission from ref. 95 . Copyright 2019 American Chemical Society. ference for $\Lambda_{1}$ lattices. Cubes with rounder edges were more able to rotate during drying and thus showed a higher degree of positional and orientational disorder.

\section{Using dispersant properties to control disorder}

An alternative route to changing the assembly behavior is to use the properties of the suspending medium. In this section we discuss the effects of including ions to screen particle interactions and controlling ambient pressure to change the rate of solvent evaporation.

\subsection{Disorder arising from electrostatic charges}

In convective self-assembly there are two classes of electrostatic interactions to consider: particle-substrate and particleparticle. Tightly packed particle assemblies result when the particles and the substrate have a repulsive interaction. In this case, the particles do not cluster near the substrate and assemble during the last stages of drying. The extra time allows the particles to accumulate and arrange into an ordered, tightly packed configuration, which minimizes surface energy. Electrostatic interactions can be controlled through the inclusion of ions in the coating solution, through both electrostatic screening of charged particles and changing the solution pH (Fig. 10). ${ }^{157}$ At higher ion concentrations, electrostatic screening reduces particle-substrate repulsion allowing the particles to be deposited before they reach the drying front. ${ }^{157}$ Rödner et al. have observed that not only particle-substrate adhesion, but also particle-particle adhesion occur at high ionic strength. ${ }^{70}$ Alternatively, by deliberately tuning solvent $\mathrm{pH}$ to increase particle surface charge, electrostatic repulsions are increased and prevent deposition and interparticle aggregation. Joshi et al. demonstrated this by adding $\left[\mathrm{OH}^{-}\right]$to a suspension of silica spheres to increase monolayer packing and order. $^{157}$

Layer thickness is also impacted by electrostatic forces. In terms of layering, particle-substrate attractive forces are overcome by particle-particle repulsive forces, leading to thinner deposits. ${ }^{175}$ When repulsive electrostatic forces between particles are weak, it is easier to pack particles together, leading to the deposition of multiple layers and longer meniscus pinning in the convective assembly regime. ${ }^{175}$

To summarize, disorder is increased by having attractive forces or charge screening, leading to particle deposition and immobility prior to the film drying.

\subsection{Disorder arising from reduced pressure}

A higher flow rate towards the drying line leads to better organization as the higher particle concentration leads to tighter packings. The evaporation rate is approximately inversely proportional to the pressure. ${ }^{176}$ Thus initially decreasing the pressure leads to an increase in the order within a colloidal film. However, if evaporation rate is too fast, some particles are deposited and immobilized before finding the lowest energy 

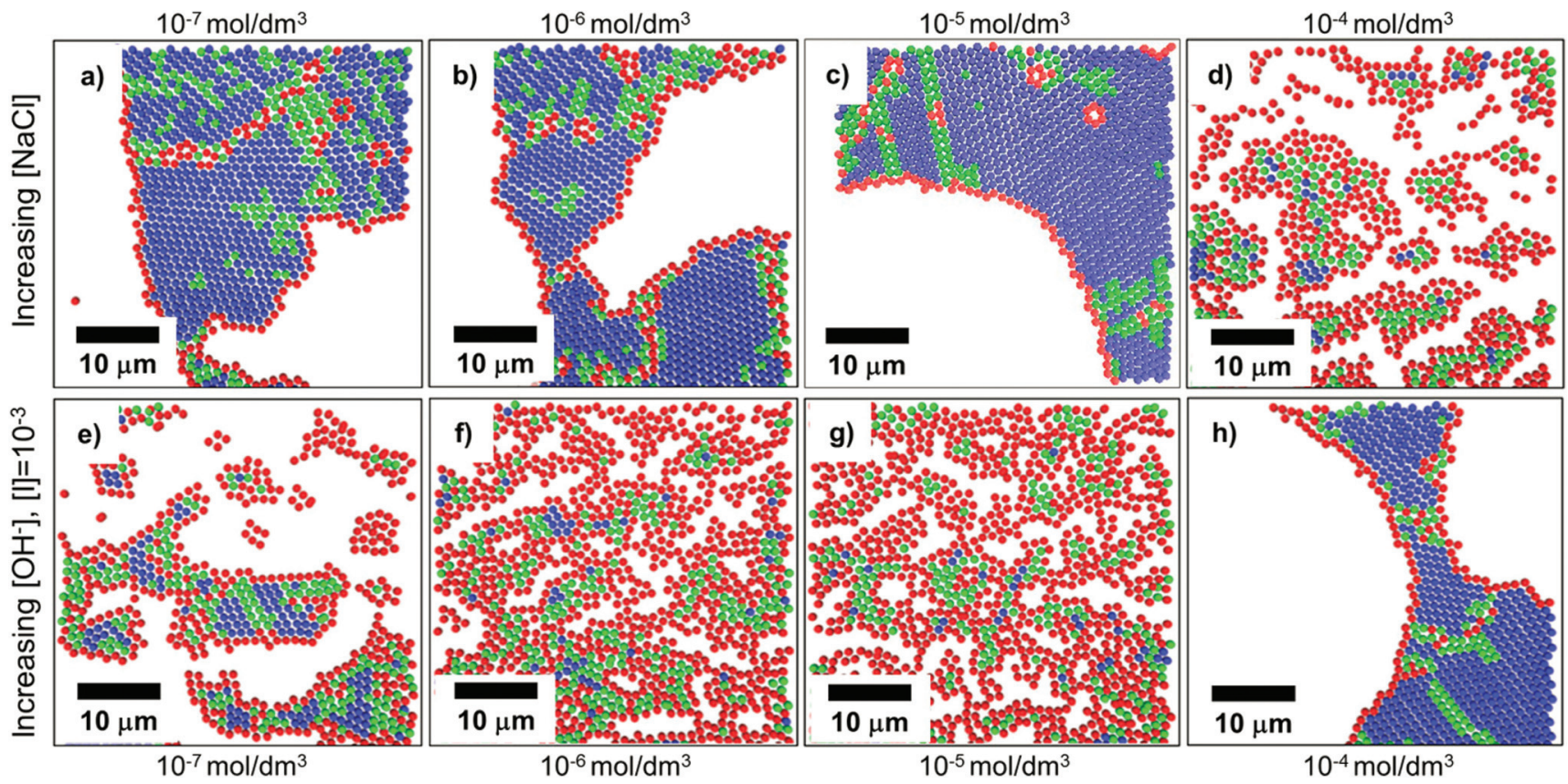

Fig. 10 Confocal scans of submonolayers, colored to indicate the number of nearest neighbors and bond order: blue indicates 6 neighbors with high bond order, green indicates 6 neighbors with medium bond order, red indicates $<6$ neighbors. (a-d) As ionic strength increases through the addition of $\mathrm{NaCl}$ to a colloidal solution, repulsive forces are shielded, and particles become more disordered due to premature deposition on the substrate. (e-h) As electrostatic charges are increased through the addition of $\left[\mathrm{OH}^{-}\right]$, order is improved. Reproduced with permission from ref. 157. Copyright 2015 American Chemical Society.

organization. ${ }^{176}$ Thus, disorder in the assembled films is highest at reduced pressure where evaporation rate is extremely fast.

\section{Using patterned substrates to control disorder}

Another option beyond modifications of the particles or the solvent is to pre-treat the substrate to control the distribution of particles. This can be achieved through purely bottom-up approaches, by which chemical patterns are generated on a substrate through self-assembly. It can also be achieved via a combination of top-down and bottom-up techniques in which a substrate is typically prepared via lithography to produce either chemical or topographical patterns. These approaches offer more precise particle positioning, and thus a higher degree of control over correlation between particles but have the limitations of lithographic approaches. Substrates produced via these means cannot be scaled up in the manner of the previous approaches due to the large amount of time required to prepare them. Hence, a trade-off currently must be made between the high degree of control offered by these approaches and their limited scalability.

\subsection{Chemically patterned substrates}

Such substrates can be broadly grouped into two different approaches used to chemically influence particle positioning: inhomogeneous chemical interactions (electrostatics, van der Waals, hydrogen bonding or even covalent bonding) and nonuniform wettability. ${ }^{177,178}$ Chemical contrast, where different chemical functionalities are pre-patterned on a substrate, creates differences in particle-substrate interactions and solvent-substrate interactions and in the evaporation rate of certain zones of the substrate. ${ }^{179}$ There are many reports of soaking chemically patterned substrates in a colloidal suspension and allowing the particles to adhere to targeted domains through particle-substrate interactions. Patterns achieved through such particle-substrate interactions lie outside the scope of this review. We encourage the interested reader to refer to other articles on the topic. ${ }^{178,180,181}$

6.1.1. Substrate-solvent interactions. Convective assembly on chemically patterned substrates is strongly affected by the interactions between the substrate and the solvent. The most common way to chemically pattern surfaces is to deposit a hydrophobic self-assembled monolayer and then to expose sections of the underlying hydrophilic substrate. Self-assembled monolayers can be patterned using photolithography, ${ }^{182,183}$ masked vapor deposition, ${ }^{184}$ selective oxidation through a mask ${ }^{185}$ or atomic force microscopy oxidation lithography. ${ }^{186}$

Selective wetting targets particle deposition on to particular domains. In the regions of particle deposition, thanks to capillary forces, highly ordered hexagonally close-packed domains self-assemble. ${ }^{182}$ In terms of substrate-solvent interactions, the change in the surface energy translates into a change in the meniscus shape. If the substrate has chemical functional- 
ities which induce a wettability contrast, the meniscus will deform between zones (Fig. 11). For polar solvents, the meniscus will be convex in hydrophilic regions and concave in hydrophobic areas. However, the meniscus can only be deformed over certain length scales. Due to the intermolecular forces between solvent molecules, the transition from a concave to a convex meniscus shape requires several micrometers. ${ }^{187}$ Certainly, the contact angles between the hydrophilic and hydrophobic zones has an impact on the resolution. However, Fustin et al. observed that the lower limits for sharp patterning were $4 \mu \mathrm{m}$ for the hydrophobic zone (non-stick zone) and $10 \mu \mathrm{m}$ for the hydrophilic zone. ${ }^{188}$ The hydrophilic-hydrophobic contrast can be accentuated by adding surfactants. ${ }^{184} \mathrm{~A}$ stable meniscus shape occurs only when the hydrophilic portion is at least twice the width of the transitional area between the two domains. Belgardt et al. found that they could overcome limits of meniscus deformability, depositing particles in a thinner hydrophilic band by dip-coating at a $45^{\circ}$ inclination. ${ }^{187}$ By changing the retraction angle, the angle of the meniscus is less steep. Thus, the differences between the contact angle of the hydrophobic and hydrophilic zones are decreased. By smoothing out this transition, the transitional area is decreased, allowing particles to be deposited in a very thin hydrophilic zone.

6.1.2. Block copolymers. The patterning of particle films and 3D structures using block co-polymers has been extensively investigated in the literature. ${ }^{189}$ Block copolymers are amphiphilic molecules typically consisting of a hydrophilic block and a hydrophobic block which will form micelles when dissolved in polar solvents. When prepared on a substrate they will self-assemble into complex patterns which segregate the opposing functionalities of the block copolymer. These typically produce either hexagonal or worm-hole assemblies (Fig. 12). Such arrangements present some disorder; in the case of the hexagonal arrangement this is present in the form of domain size polydispersity and variable inter domain spacings. Whereas the worm-hole organization is a complex pattern of branching linear structures of approximately constant width which form an entangled network covering the entire surface. Such polymers offer a route to pattern substrates without the use of lithography, and hence represent a fully bottom-up approach to the preparation of patterned substrates.

The block copolymer templated approach is particularly interesting for nanoparticles smaller than the block copolymer domains. Nanoparticles are suspended in a block copolymer solution and then formed into a thin film through solvent evaporation combined with either spin-coating, film casting, drop-casting, or rarely, dip-coating. In the framework of this review, we will discuss patterns formed through one-pot assembly. A more in-depth discussion of such materials can be found in other reviews. ${ }^{189,190}$ Block copolymer and nanoparticle co-assembly leads to the nanoparticles being positioned in a hydrophobic block, a hydrophilic block, or at the interface between the two. The nanoparticles can be designed to have a preferential affinity for one of the blocks (Fig. 13). Surface chemistry is not the sole factor influencing particle placement. There is an entropic influence related to the rela-

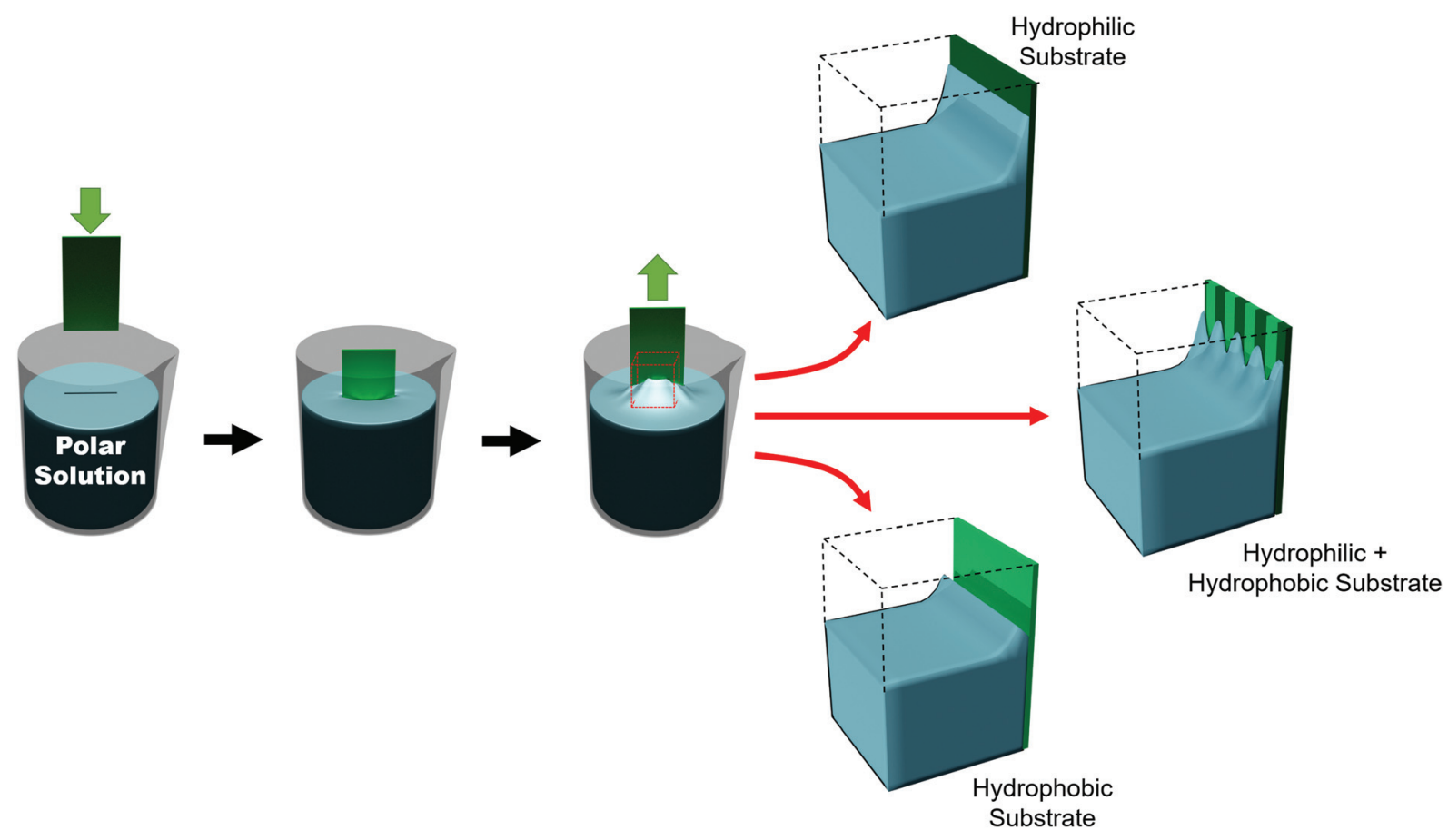

Fig. 11 Meniscus of a polar solution on a hydrophilic, a hydrophobic, and a patterned hydrophilic/hydrophobic substrate. 
(a)

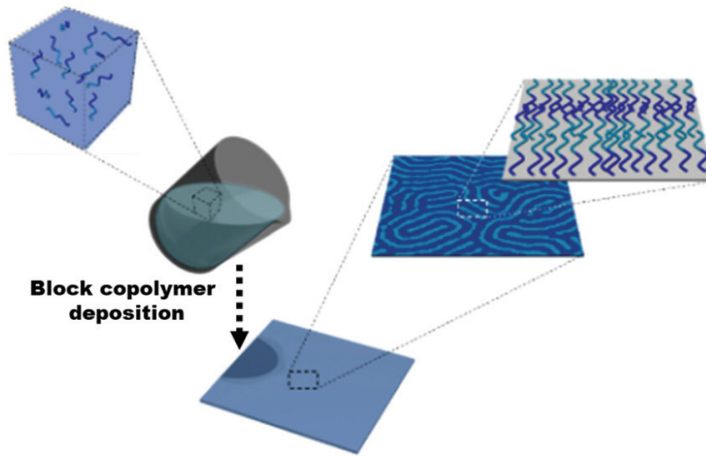

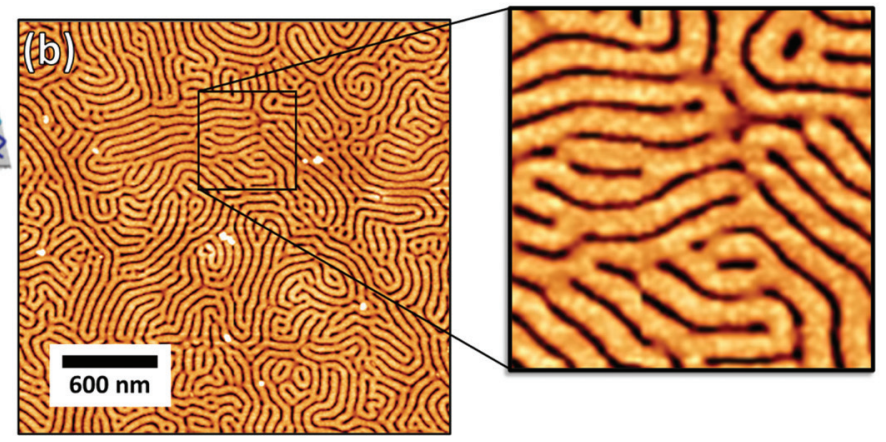

Fig. 12 (a) Block copolymer deposition and self-assembly on a substrate. (b) AFM of a deposited block copolymer (PS(102k)-b-P2VP(97k)) on a silicon substrate. Random organization of domains having constant width.

(a)

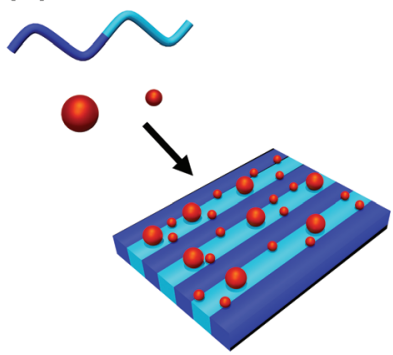

(c)

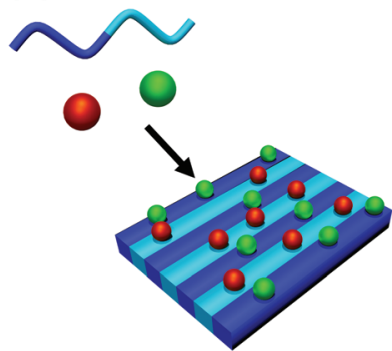

(d)

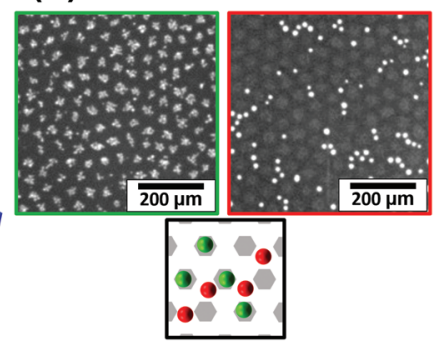

Fig. 13 Selective deposition of particles of different ( $a$ and b) size and (c and d) nature on a self-assembled block copolymer film. (b) TEM image of a binary mixture of two particle sizes $(D<3 \mathrm{~nm}$ and $D>5 \mathrm{~nm})$ deposited on a PS-PEP-PS triblock copolymer; smaller particles migrate to domain interfaces and larger particles tend to stay inside domains. (d) SEM images of silver nanoparticles on [PS(57k)-b-P4VP (18k)] (red/right) and gold nanoparticles on [PS(57k)-b-P4VP(18k)] (green left); affinity of gold for P4VP and of silver for PS. (b) Reproduced with permission from ref. 192. Copyright 2006 American Chemical Society. (d) Reproduced with permission from ref. 193. Copyright 2013 John Wiley \& Sons.

tive size of the nanoparticle and the block copolymer domains (Fig. 13). ${ }^{191,192}$ Smaller particles migrate to the interface, whereas larger particles position themselves fully within the corresponding block. By regulating the size and surface chemistry of these patterns it is possible to achieve targeted spatial control.

Evaporatively driven convection also impacts particle placement. Li et al. compared block copolymer-gold nanoparticle
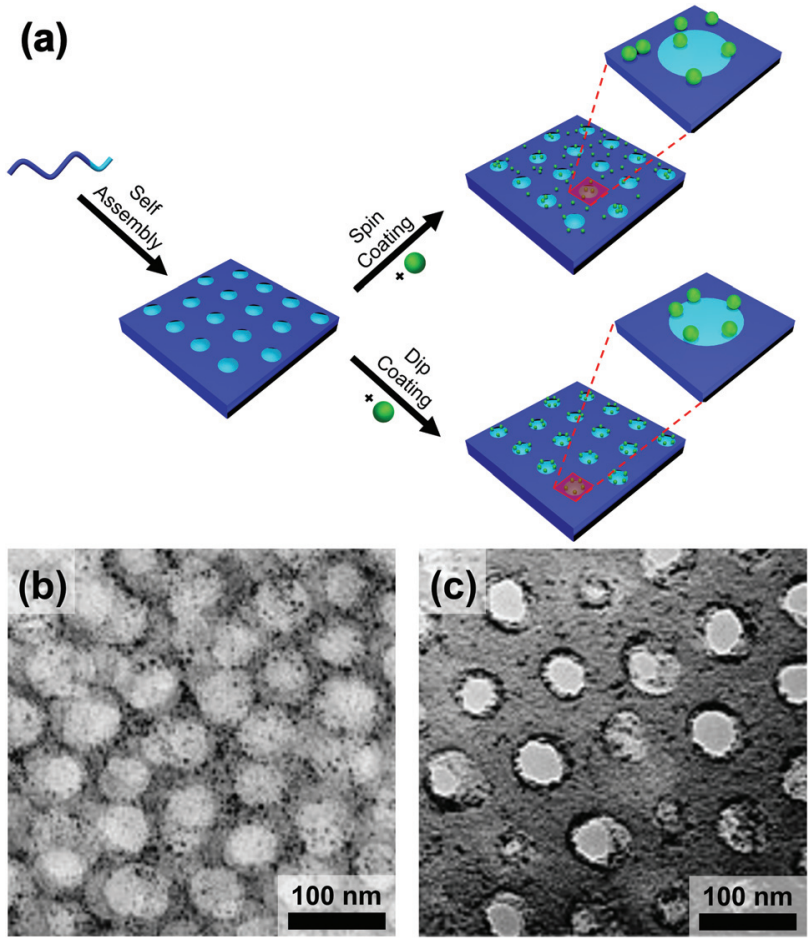

Fig. 14 (a) Schematic of the block copolymer templating approach via spin-coating and dip-coating. TEM micrographs of thin films of blockcopolymer (PS- $b$-P2VP)/gold nanoparticles prepared by (b) spin-coating and (c) dip-coating. Deposition by spin-coating leads to a random deposition of particles when deposition by dip-coating leads to a confinement of the particles at the interface between two domains. (b) \& (c) Reproduced with permission by ref. 194. Copyright 2008 John Wiley $\&$ Sons.

thin films prepared by both spin- and dip-coating (Fig. 14). ${ }^{194}$ The greater time elapsed during dip-coating allowed the micelles and nanoparticles to configure themselves on the substrate, resulting in particle migration to the interface. Control over the evaporation rate through the factors discussed above, particularly liquid film thickness and atmospheric conditions will regulate convective flux and thus kinetically control the rate of particle placement. 
In this section, we discussed particle assembly on chemically patterned substrates and the self-assembly of nanoparticles using block copolymers. Chemical contrast influenced the shape and separation of the domains, and the quantity of particles within them. Disordered chemical contrast can be designed using top-down patterning techniques, or it can form through bottom-up self-assembly of patterns. Chemical patterning disrupts the continuity of particle deposition, allowing stopbands to be tailored. ${ }^{182}$

With additional complexity in the forces acting on the colloidal solution, the particles demonstrate unusual, and less ordered, deposition behavior. For instance, wettability contrast destabilizes the shape of the meniscus and changes the evaporation rate along the drying line. This is apparent when using motif bands parallel to the meniscus, which cause the contact angle to vary as the substrate is withdrawn acting in opposition to stick-slip behavior. Also, the high degree of curvature in some motifs prevents the close packing of spheres leading to the formation of lattice defects. In summary, disorder is generated, but moderated over large length scales via chemical patterning.

\subsection{Topographically patterned substrates}

An approach that offers greater control over particle distribution than chemical patterning and non-templated techniques is that of particle deposition onto topographically patterned substrates. Such substrates are typically produced through the modification of a polymer layer applied above a flat substrate through top-down techniques such as lithography. Lithography-free protocols also exist, including wrinkles induced through repeated plastic deformation ${ }^{197}$ and the grooves of optical discs. ${ }^{198}$ Normally these substrates feature depressions or trenches with at least one dimension of similar magnitude to the particle. Particles can then be directed over the substrate in high concentration and the particles are trapped within the depressions through capillary action.
While convection is the process transporting particles to the meniscus, and thus to the traps, assembly within the traps themselves is driven primarily by capillary forces (Fig. 15a). Particles remain mobile after the triple contact line has receded beyond the physical trap allowing further reorganization. Mobility within the traps is dictated by the strength of the capillary forces between particles and between the trap and the particles. Hence particle organization within physical traps is independent of whether convective flow is used. Thus, while discussion in this section is primarily focused on topographically patterned substrates used in combination with convective assembly, some studies have been included that feature applicable results, which do not use convective assembly. We present examples organized into four classes of physical pattern.

6.2.1. Traps larger than the particle - single particles. The simplest examples of these traps are pits slightly larger in width than the particles of interest. The capillary forces on particles, as they are directed over such pits, trap single particles at precise locations on the substrate. In the literature, these have mostly been realized as a protocol to produce highly ordered non-close packed arrays of particles using a range of morphologies, including spheres, ${ }^{101,199-203}$ rods, ${ }^{195,204}$ cubes, ${ }^{205-207}$ and plates. ${ }^{207}$ The greatest potential of this technique, regarding the assembly of disordered monolayers, is that particles can be assembled into any arbitrary two-dimensional spatial distribution. These include complex designs with precision at the single pixel level (Fig. 15b). ${ }^{195,196,202,204,208}$ Hence systems can be devised and reliably produced with known levels of correlation and particle density. Beyond this the relative orientations of anisotropic particles can be similarly controlled at the individual particle level giving precise control over both spatial and orientational correlation (Fig. 15c). ${ }^{204,207}$ Templates patterned in such a manner offer a direct route to study the effect of disordered assemblies of particles in a highly controlled environment, although such studies have not yet been performed.

6.2.2. Traps much wider than the particle - clusters and bands. Increasing the size of the traps to accommodate mul- (a)

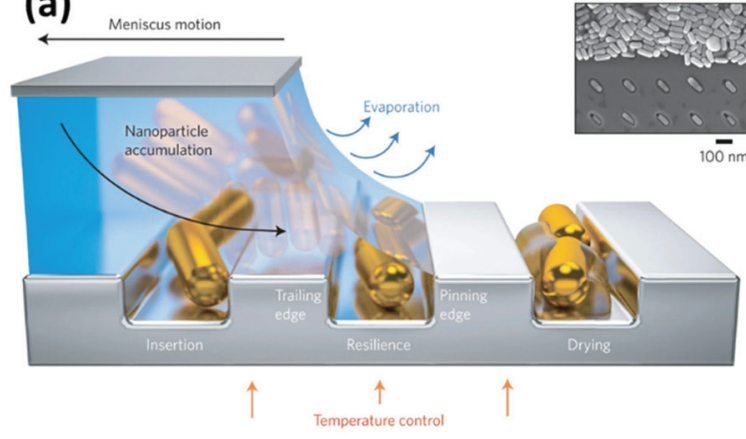

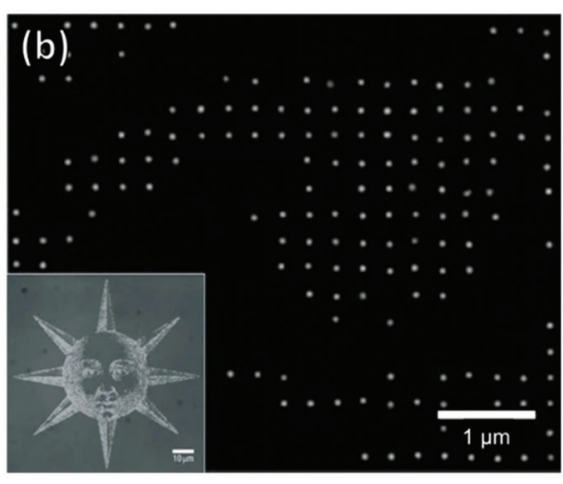
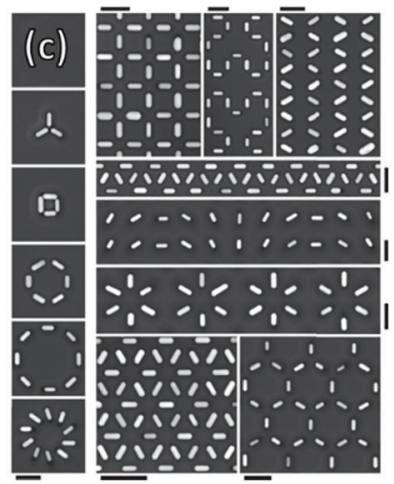

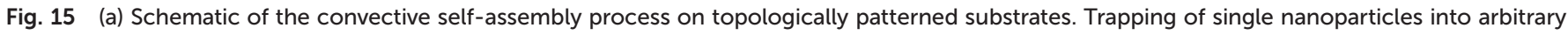

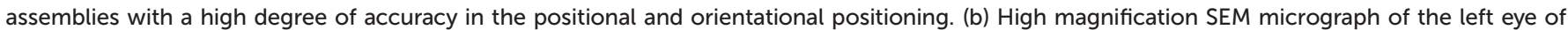

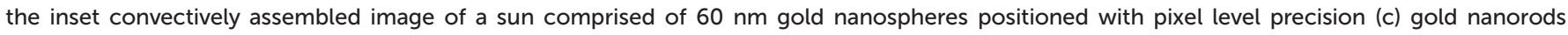

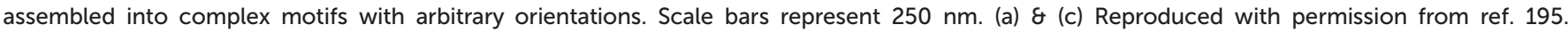
Copyright 2017 Nature Publishing Group. (b) Reproduced with permission from ref. 196. Copyright 2007 Nature Publishing Group. 
tiple particles allows for a hierarchy of particle organization, with control over the spacing between regions of particles and the packing within them. So far these have largely manifested themselves in the literature as either two-dimensional clusters ${ }^{209-213}$ or striped domains. ${ }^{198,214-216}$ Key to controlling the internal disorder of these regions is the ratio of the size of the trap to the dimensions of a fully compressed lattice. By deliberately introducing a mismatch between these, voids are created, decreasing packing fraction and introducing internal disorder. Disorder is higher for small particles $(\lesssim 10 \mathrm{~nm})$ because forces such a Brownian motion act against capillary trapping, and the resolution limits of lithography makes it difficult to closely match the size of the traps to the particles. $^{217,218}$

Most literature reports of topographically-templated clusters consist of arrays of well-separated, uniform clusters. ${ }^{202,209-213,219}$ Greybush et al. found that increasing the width of such traps beyond seven particles led to increasing disorder in the clusters. ${ }^{212}$ They attributed this to polydispersity, which prevented regular close-packing. The increase in disorder was visible in the optical spectra of individual clusters with complex features and blue-shifted peaks relative to closepacked clusters of monodisperse gold nanospheres.

Shillingford et al. were able to create hexagonally closepacked, square, and disordered packings within arrays of square traps by changing particle volume fraction, and tuning the depth and resolution of the traps. ${ }^{222}$ Disordered packings were observed at low volume fractions, with square and hexagonally close-packed organization at higher fractions. Amorphous monolayers could be obtained using poorly resolved traps featuring kinks and breaks which disrupted lattice order. In the case of linear bands, confinement applied consistently along a single axis results in linear structures, ranging from single particle columns to several particle thick bands. ${ }^{198,214,215,219,223,224}$ The disorder within these bands is highly sensitive to the width of the channel, $\Lambda$, versus the width of a close packed lattice. Disorder is obtained by deviating from a set of specific widths, which can be calculated from the relationship $\Lambda=D[1+\sqrt{3 / 2}(n-1)]$, where $D$ is the particle diameter, and $n$ is the number of particle columns in the channel, as seen in Fig. 16. As polydispersity and particle mobility must be accounted for, traps must be slightly wider than these widths to produce close-packed lattices. A small additional deviation results in the appearance of line defects (Fig. 16a and b). ${ }^{219}$ Conversely strong deviations from these ideal values leads to disordered monolayers (Fig. 16c-e). ${ }^{219,223}$ The packing fraction in the assembled regions of monolayer serves as a good figure of merit to quantify disorder as a function of channel width (Fig. 16f). ${ }^{223}$

6.2.3. Patterns with features smaller than the particles. Traps can be made smallers than the particle of interest. Capillary forces will trap a particle without it being fully enclosed, allowing for particles to contact each other between traps. This has important implications for the packing of neighboring particles. These patterns can consist of both holes $^{226,227}$ and stripes. ${ }^{220,228}$ In both cases disorder occurs by

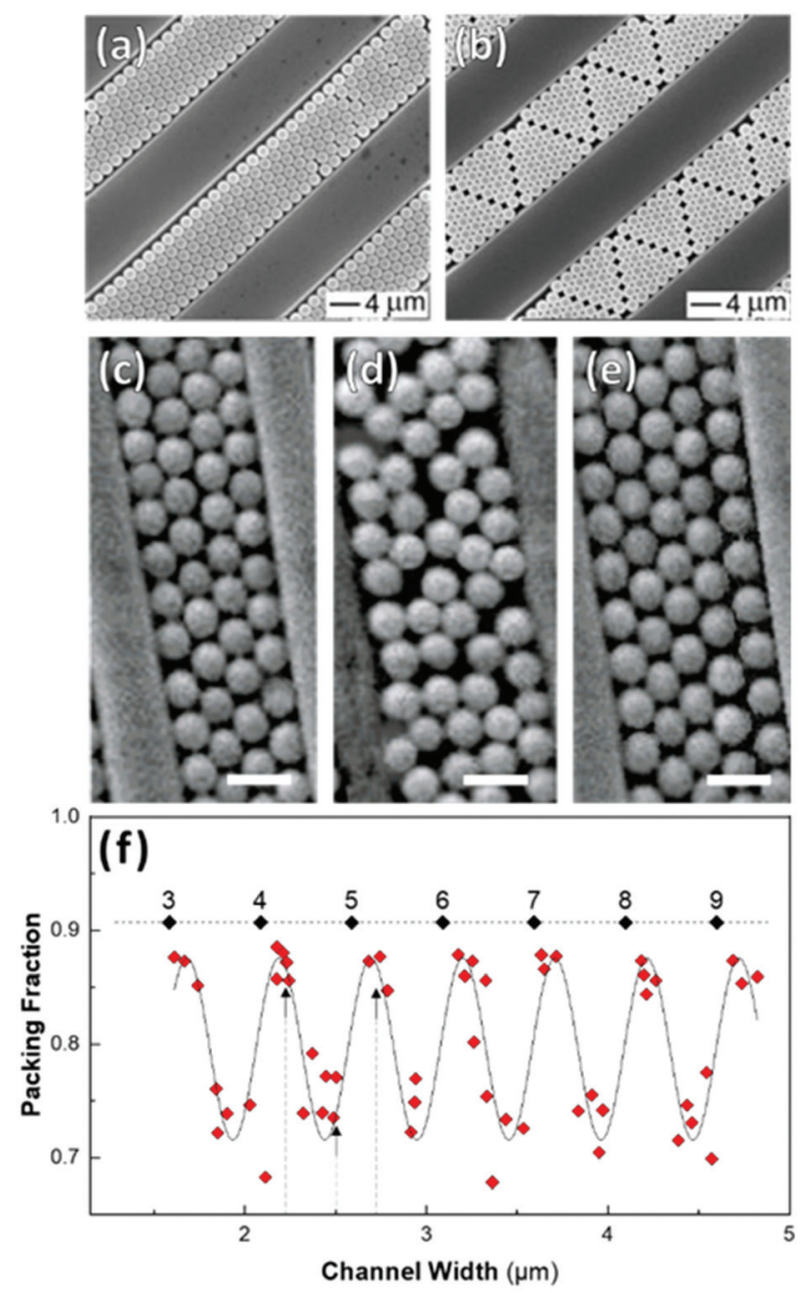

Fig. 16 Effect of confinement on particle organization. (a \& b) Deviation in channel width, from a value commensurate with a closepacked lattice, induces the formation of linear defects. (c)-(e) Disordered assembly within the channel is caused by intermediate channel widths between those compatible with a close-packed lattice (as seen in (d)). Scale bar represents $1 \mu \mathrm{m}$ (f) Monolayer packing fraction as a function of channel width showing the decrease in packing density when widths incommensurate with close packing are used. Dashed line $=0.9069$ ( $\max$ packing fraction). Labels are the number of particle columns. (a) \& (b) Reproduced with permission from ref. 219. Copyright 2003 John Wiley $\&$ Sons. (c) - (f) adapted with permission from ref. 223. Copyright 2002 John Wiley \& Sons.

introducing a mismatch between the positioning of the traps and the particle centers of a close-packed lattice. These distances are easy to calculate for hexagonally close-packed lattices (inter-trap distance, $\Lambda=\sqrt{ } 3 D / 2)$ and square lattices $(\Lambda=$ $\sqrt{ } 2 D$ ). Any deviation from these values will result in the formation of voids and a disordered lattice.

In the case of striped patterns, values of $\Lambda<\sqrt{ } 3 D / 2$ result in an increasingly square lattice with ever larger voids down to a critical value of $\Lambda=\sqrt{ } 2 D$, below which it is no longer possible to populate all traps on the substrate (Fig. 17a-c). Values of $\Lambda>$ $\sqrt{3 D / 2}$ result in decreasing overlap between particles trapped 

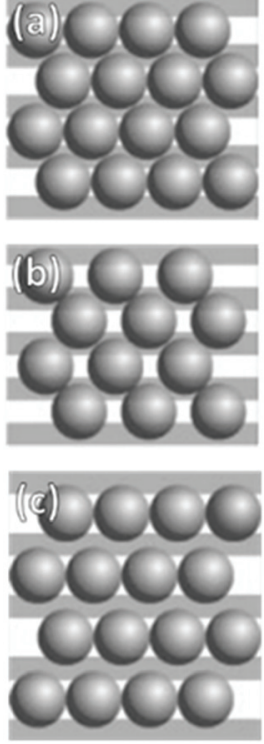
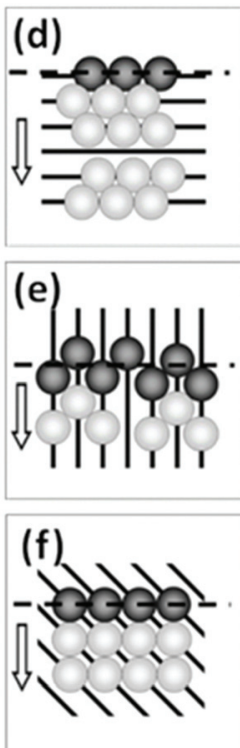
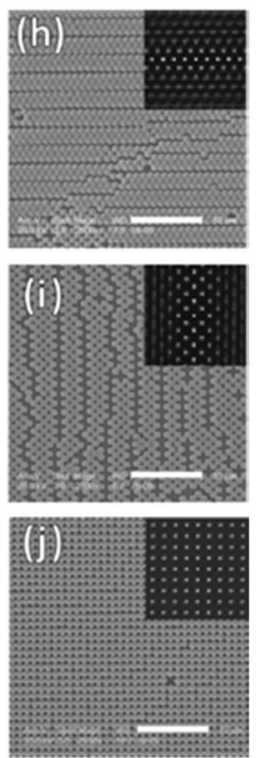

Fig. 17 Particle monolayers templated using patterns smaller than the particle. (a)-(c) Varying inter-trap distance, $\Lambda$ leads to changes in particle organization i.e. (a) perfect matching $(\Lambda=\sqrt{ } 3 D / 2)$, (b) $\Lambda<\sqrt{ } 3 D / 2$, and (c) $\Lambda>D$. (d) $-(\mathrm{j})$ Trap orientation relative the meniscus impacts packing structures. Arrows indicate meniscus travel direction. (d) Parallel traps cause hexagonal close-packing with parallel defects. (e) Perpendicular traps cause square packing with perpendicular defects. (f) $45^{\circ}$ traps cause near-perfect square lattice. (h)-(j) SEM micrographs of (d)-(f) with inset 2D autocorrelation graphs. Scale bars represent $10 \mu \mathrm{m}$. (a)-(c) Reproduced with permission from ref. 220. Copyright 2004 John Wiley \& Sons. (d)-(i) Reproduced with permission from ref. 221. Copyright 2008 John Wiley \& Sons.
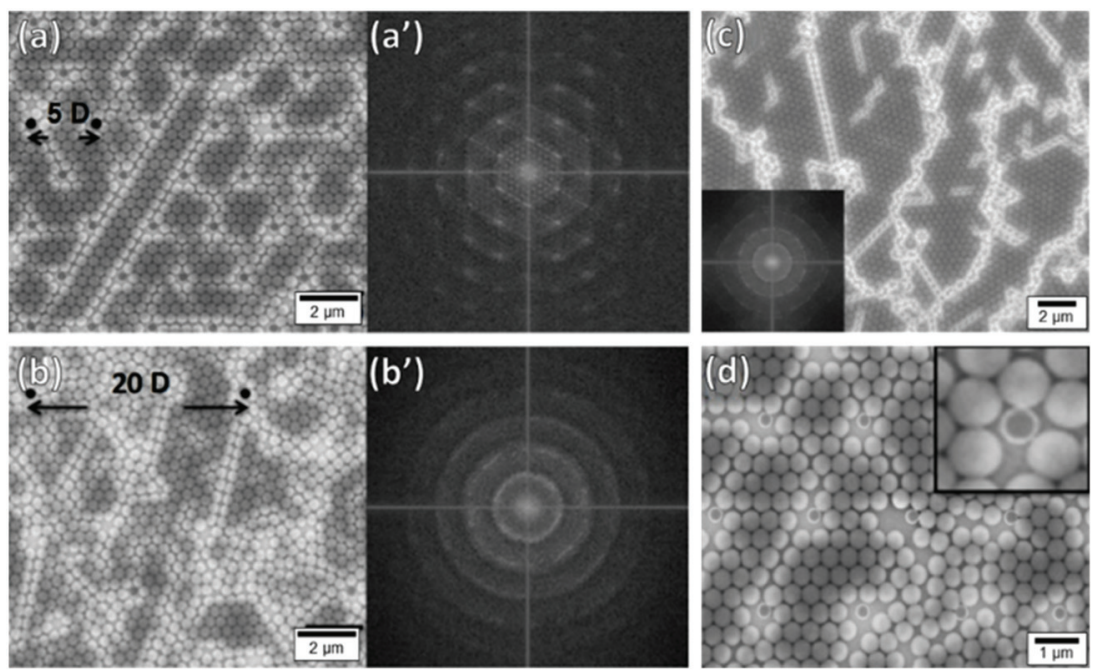

Fig. 18 Particle monolayers templated using substrates patterned with pillars showing the impact of pillar spacing and mismatching pillar size (a) Good size matching between pillars and particles with small interpillar spacings $(5 D)$ produce hexagonal close-packed lattices with ordered 2D Fourier transforms (a'). (b) Good size matching between pillars and particles with large interpillar spacings (20D) produce more disordered lattices with rings in the 2D Fourier transforms ( $\left.b^{\prime}\right)$. (c) Template free deposition produces poly disordered lattices with many distinct domains. (d) Using smaller pillars $(296 \mathrm{~nm})$ leads to the formation of lattice defects. Figure reproduced with permission from ref. 225 . Copyright 2014 Beilstein-Institut. 

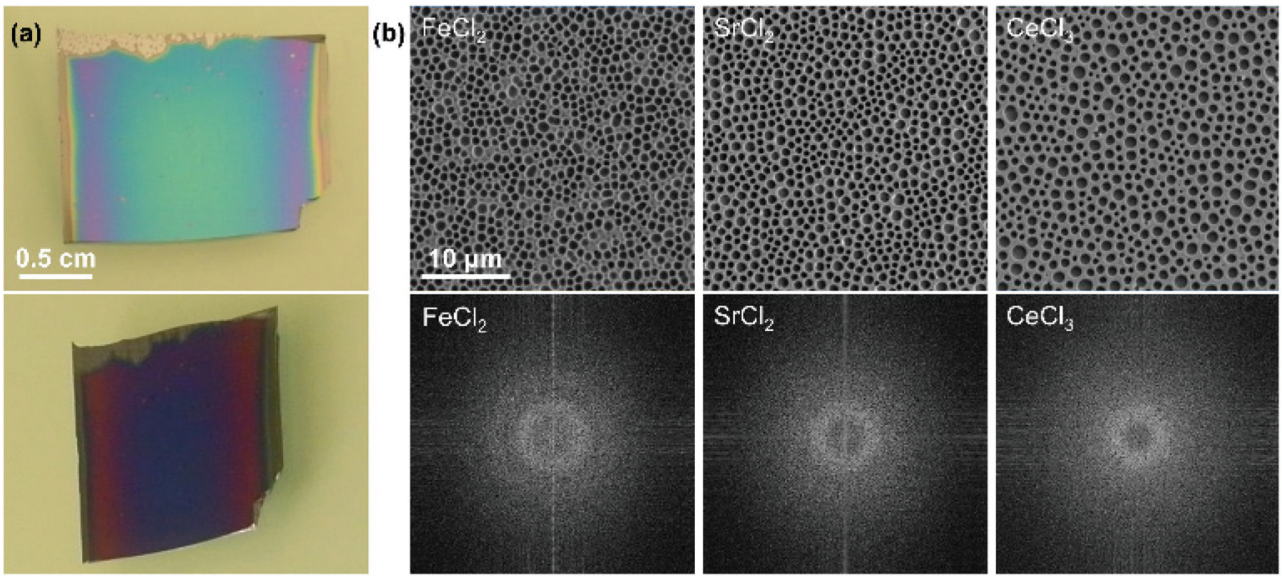

Fig. 19 (a) Photographs of a microperforated film at two different angles. (b) SEM micrographs of macroperforations made by using different salts and their fast Fourier transforms showing differences in the degree of order and the distance between perforations. The scale is the same for all micrographs. Figure adapted with permission from ref. 232. Copyright 2014 John Wiley \& Sons.

physical obstructions to disrupt lattice order is a highly effective method to control disorder within convectively assembled films.

\section{Perspectives}

Both dip-coating and drag-coating offer additional parameters to those discussed above, which have yet to be fully investigated for their effect on disorder in colloidal films. Specifically, solvent properties, such as viscosity and surface tension, will have significant consequences on particle assembly. Solvent properties are apt to create disorder on scales larger than the particle, for instance generating irregular gaps and non-linear banding. This type of patterning can be caused due to Marangoni flows.

Of all the systems discussed, topographically patterned substrates offer the greatest degree of control over spatial and rotational disorder. However, most topographically patterned substrates use lithographic techniques that limit their scalability. Introducing a pattern using nanoimprint lithography offers a route to bulk production of substrates with tailored disorder. The effects of disorder achieved in these systems on their optical properties has not been investigated thoroughly in the literature. Clearly, there is great potential in these techniques for creating systems with near-complete control of the spatial and rotational disorder. This would allow us to fully explore the impact of disorder on the optical properties of twodimensional colloidal assemblies.

Another frontier that remains largely unexplored is hierarchical disorder. For instance, in binary mixtures of particles, disorder can occur in particles of one size, while not impacting the crystallinity of a second population of particles, allowing interactions with waves to be highly tuned.

Inexistant to date, is the use of non-flat surfaces to influence disorder in convective assembly. Here substrate curvature, applied post-synthetically, could be used to introduce lattice defects and vary correlation distance within assembled films.

Post-synthetic techniques using mechanical forces, including among others, strain, shear, and scratching, are promising routes to control disorder. ${ }^{231}$ Flexible substrates can potentially produce surfaces over which correlation distances could be dynamically changed.

In this review we have extensively discussed particle assemblies, but other structures can be created using evaporative processes. Monolayer perforated films can be made with interesting angle-dependent appearances (Fig. 19) ${ }^{232}$ We previously produced such films, showing how salt and surfactant concentration, relative humidity and withdrawal speed all impacted perforation density and size, and thus film appearance. The degree of disorder in the macroperforations was affected by the type of salt used (Fig. 19), certainly other parameters could also be explored in terms of their impact on order regulating dewetting. By comparison, topographical patterning offers highly precise control through capillary forces in confined spaces. Of particular interest, is the potential of topological patterning to manage disorder in non-close packed systems, where both rotational and positional control can be exerted with high accuracy.

\section{Conclusions}

Currently, most examples of disorder in convectively assembled systems appear in studies which aim to create organized close-packed particle assemblies. Historically disorder has been seen as undesirable, more recently however, a controllable degree of disorder has proved advantageous for some applications, particularly in optics. We can reverse engineer the lessons learned from optimizing order in colloidal films to find the parameters impacting disorder. In this review, we have presented the main contributions in terms of the particle, solvent, and substrate properties affecting particle organization during convective assembly. 
To have a good control over particle placement, many of the same considerations are needed to achieve disorder as those required for obtaining crystallinity: excellent wettability, strongly repulsive interparticle and particle-substrate forces, high evaporative flux, and good particle mobility. Conversely, polydispersity in size and shape, a mismatch between particles and templates, and polymodal particle populations, which are harmful to close packing, can be used to tailor the degree of disorder.

In general, drag-coating gives better control of particle assembly than dip-coating, but is less amenable to coating curved surfaces and large areas. Spheres are the easiest objects to assemble, as orientational disorder does not need to be considered, and preferential packing between certain faces does not occur. However, irrespective of shape, disorder can be managed.

We can target very specific particle distributions through substrate patterning. During convective assembly, chemical patterning gives approximate control within specific regions by regulating dewetting. By comparison, topographical patterning offers highly precise control through capillary forces in confined spaces. Of particular interest, is the potential of topological patterning to manage disorder in non-close packed systems, where both rotational and positional control can be exerted with high accuracy.

\section{Author contributions}

$\mathrm{LR}, \mathrm{AH}, \mathrm{KV}$, and GLD were involved in writing of the original draft and visualization. PL, ED, MTD, KV, and GLD were involved in supervision and funding acquisition. LR and GLD were responsible for project administration. All authors were involved in the reviewing and editing of the final manuscript.

\section{Conflicts of interest}

The authors declare no conflicts of interest.

\section{Acknowledgements}

LR and GLD received funding for this work from the European Research Council (ERC) under European Union's Horizon 2020 research and innovation programme (grant no. 948319). LR, GLD, ED, and MTD received financial support from the Conseil Régional d'Aquitaine and the LabEx AMADEus (grant no. ANR-10-LABX-42) in the framework of IdEx Bordeaux (grant no. ANR-10-IDEX-03-02) managed by the National Agency for Research. AH, PL, ED, MTD, KV, and GLD received financial support from the Centre National de la Recherche Scientifique (CNRS) through the MITI interdisciplinary programs and from the ANR (grant no. ANR-19-CE09-0014). AH also received support from the Light S\&T Graduate Program (PIA3 Investment for the Future Program, grant no. ANR-17EURE-0027).

\section{References}

1 V. Tuchin, Tissue Optics: Light Scattering Methods and Instruments for Medical Diagnosis, SPIE Press, 2015.

2 M. I. Mishchenko, J. W. Hovenier and L. D. Travis, Light Scattering by Nonspherical Particles: Theory, Measurements, and Applications, Elsevier Science, 1999.

3 D. S. Wiersma, Nat. Photonics, 2013, 7, 188.

4 R. Carminati and J. C. Schotland, Principles of Scattering and Transport of Light, Cambridge University Press, 2021.

5 M. Florescu, S. Torquato and P. J. Steinhardt, Proc. Natl. Acad. Sci. U. S. A., 2009, 106, 20658.

6 L. F. Rojas-Ochoa, J. M. Mendez-Alcaraz, J. J. Sáenz, P. Schurtenberger and F. Scheffold, Phys. Rev. Lett., 2004, 93, 073903.

7 F. Riboli, N. Caselli, S. Vignolini, F. Intonti, K. Vynck, P. Barthelemy, A. Gerardino, L. Balet, L. H. Li, A. Fiore, M. Gurioli and D. S. Wiersma, Nat. Mater., 2014, 13, 720.

8 H. Noh, J.-K. Yang, S. F. Liew, M. J. Rooks, G. S. Solomon and H. Cao, Phys. Rev. Lett., 2011, 106, 183901.

9 L. Sapienza, H. Thyrrestrup, S. Stobbe, P. D. Garcia, S. Smolka and P. Lodahl, Science, 2010, 327, 1352.

10 S. Yu, C.-W. Qiu, Y. Chong, S. Torquato and N. Park, Nat. Rev. Mater., 2021, 6, 226.

11 M. Rothammer, C. Zollfrank, K. Busch and G. von Freymann, Adv. Opt. Mater., 2021, 9, 2100787.

12 K. Vynck, R. Pierrat, R. Carminati, L. S. Froufe-Pérez, F. Scheffold, R. Sapienza, S. Vignolini and J. J. Sáenz, 2021, arXiv:2106.13892.

13 H. Brumberger, Modern Aspects of Small-Angle Scattering, Springer, Netherlands, 2013.

14 S. G. Romanov, S. Orlov, D. Ploss, C. K. Weiss, N. Vogel and U. Peschel, Sci. Rep., 2016, 6, 27264.

15 R. O. Prum and R. Torres, J. Exp. Biol., 2003, 206, 2409.

16 J. Haberko, L. S. Froufe-Pérez and F. Scheffold, Nat. Commun., 2020, 11, 4867.

17 L. Tsang, J. A. Kong and K. H. Ding, Scattering of Electromagnetic Waves: Theories and Applications, Wiley, 2004.

18 C. Battaglia, K. Söderström, J. Escarré, F.-J. Haug, D. Dominé, P. Cuony, M. Boccard, G. Bugnon, C. Denizot, M. Despeisse, A. Feltrin and C. Ballif, Appl. Phys. Lett., 2010, 96, 213504.

19 C. Rockstuhl, S. Fahr, K. Bittkau, T. Beckers, R. Carius, F.-J. Haug, T. Söderström, C. Ballif and F. Lederer, Opt. Express, 2010, 18, A335.

20 P. Kowalczewski, M. Liscidini and L. C. Andreani, Opt. Lett., 2012, 37, 4868.

21 Y. J. Donie, D. Theobald, S. Moghadamzadeh, A. Mertens, I. M. Hossain, U. W. Paetzold, U. Lemmer and G. Gomard, Adv. Opt. Mater., 2021, 9, 2001610.

22 M. E. Stewart, C. R. Anderton, L. B. Thompson, J. Maria, S. K. Gray, J. A. Rogers and R. G. Nuzzo, Chem. Rev., 2008, 108, 494.

23 R. Brunner, O. Sandfuchs, C. Pacholski, C. Morhard and J. Spatz, Laser Photonics Rev., 2012, 6, 641. 
24 D. Bedeaux and J. Vlieger, Optical Properties of Surfaces, Imperial College Press, 2004.

25 S. Grésillon, L. Aigouy, A. C. Boccara, J. C. Rivoal, X. Quelin, C. Desmarest, P. Gadenne, V. A. Shubin, A. K. Sarychev and V. M. Shalaev, Phys. Rev. Lett., 1999, 82, 4520.

26 A. Moreau, C. Ciracì, J. J. Mock, R. T. Hill, Q. Wang, B. J. Wiley, A. Chilkoti and D. R. Smith, Nature, 2012, 492, 86.

27 P. R. Wray and H. A. Atwater, ACS Photonics, 2020, 7, 2105.

28 A. Jouanin, J. P. Hugonin and P. Lalanne, Adv. Funct. Mater., 2016, 26, 6215-6223.

29 M. Albooyeh, S. Kruk, C. Menzel, C. Helgert, M. Kroll, A. Krysinski, M. Decker, D. N. Neshev, T. Pertsch, C. Etrich, C. Rockstuhl, S. A. Tretyakov, C. R. Simovski and Y. S. Kivshar, Sci. Rep., 2014, 4, 4484.

30 M. Jang, Y. Horie, A. Shibukawa, J. Brake, Y. Liu, S. M. Kamali, A. Arbabi, H. Ruan, A. Faraon and C. Yang, Nat. Photonics, 2018, 12, 84.

31 M. Dupré, L. Hsu and B. Kanté, Sci. Rep., 2018, 8, 7162.

32 H. Bertin, Y. Brûlé, G. Magno, T. Lopez, P. Gogol, L. Pradere, B. Gralak, D. Barat, G. Demésy and B. Dagens, ACS Photonics, 2018, 5, 2661.

33 E. El Shamy, J. Jaeck, R. Haïdar and P. Bouchon, Appl. Phys. Lett., 2019, 115, 041103.

34 H. Zhang, Q. Cheng, H. Chu, O. Christogeorgos, W. Wu and Y. Hao, Appl. Phys. Lett., 2021, 118, 101601.

35 P. M. Piechulla, B. Fuhrmann, E. Slivina, C. Rockstuhl, R. B. Wehrspohn and A. N. Sprafke, Adv. Opt. Mater., 2021, 9, 2100186.

36 F. Sterl, E. Herkert, S. Both, T. Weiss and H. Giessen, ACS Nano, 2021, 15, 10318.

37 P. Mao, C. Liu, X. Li, M. Liu, Q. Chen, M. Han, S. A. Maier, E. H. Sargent and S. Zhang, Light: Sci. Appl., 2021, 10, 180.

38 F. Kretschmer, S. Mühlig, S. Hoeppener, A. Winter, M. D. Hager, C. Rockstuhl, T. Pertsch and U. S. Schubert, Part. Part. Syst. Charact., 2014, 31, 721-744.

39 M. L. De Marco, S. Semlali, B. A. Korgel, P. Barois, G. L. Drisko and C. Aymonier, Angew. Chem., Int. Ed., 2018, 57, 4478-4498.

40 X. Lu, M. Rycenga, S. E. Skrabalak, B. Wiley and Y. Xia, Annu. Rev. Phys. Chem., 2009, 60, 167-192.

41 Z. Wu, S. Yang and W. Wu, Nanoscale, 2016, 8, 1237-1259.

42 S. Kumar and T. Nann, Small, 2006, 2, 316-329.

43 D. A. Keen and A. L. Goodwin, Nature, 2015, 521, 303-309.

44 P. M. Ossi, Disordered Materials - An Introduction, SpringerVerlag, Berlin Heidelberg, 2006.

45 V. Lotito and T. Zambelli, Adv. Colloid Interface Sci., 2017, 246, 217.

46 V. Lotito and T. Zambelli, Adv. Colloid Interface Sci., 2020, 284, 102252.

47 C. P. Martin, M. O. Blunt, E. Pauliac-Vaujour, A. Stannard, P. Moriarty, I. Vancea and U. Thiele, Phys. Rev. Lett., 2007, 99, 116103.

48 E. Armstrong, W. Khunsin, M. Osiak, M. Blömker, C. M. S. Torres and C. O’Dwyer, Small, 2014, 10, 1895.
49 J. C. Earnshaw and D. J. Robinson, J. Phys.: Condens. Matter, 1990, 2, 9199.

50 H. J. Schöpe, J. Phys.: Condens. Matter, 2003, 15, L533.

51 I. M. Krieger and F. M. O’Neill, J. Am. Chem. Soc., 1968, 90, 3114.

52 S. A. Asher, J. M. Weissman, A. Tikhonov, R. D. Coalson and R. Kesavamoorthy, Phys. Rev. E, 2004, 69, 066619.

53 A. Tikhonov, R. D. Coalson and S. A. Asher, Phys. Rev. B: Condens. Matter Mater. Phys., 2008, 77, 235404.

54 C. Deleuze, B. Sarrat, F. Ehrenfeld, S. Perquis, C. Derail and L. Billon, Phys. Chem. Chem. Phys., 2011, 13, 10681.

55 M. Klokkenburg, B. H. Erné, A. Wiedenmann, A. V. Petukhov and A. P. Philipse, Phys. Rev. E, 2007, 75, 051408.

56 A. Wiedenmann, M. Kammel, A. Heinemann and U. Keiderling, J. Phys.: Condens. Matter, 2006, 18, S2713.

57 J. Li, B. Cabane, M. Sztucki, J. Gummel and L. Goehring, Langmuir, 2012, 28, 200.

58 M. Megens, C. M. van Kats, P. Bösecke and W. L. Vos, Langmuir, 1997, 13, 6120.

59 P. Siffalovic, E. Majkova, L. Chitu, M. Jergel, S. Luby, A. Satka and S. V. Roth, Phys. Rev. B: Condens. Matter Mater. Phys., 2007, 76, 195432.

60 A. C. Y. Liu, R. F. Tabor, M. D. de Jonge, S. T. Mudie and T. C. Petersen, Proc. Natl. Acad. Sci. U. S. A., 2017, 114, 10344.

61 L. Wu, X. Wang, G. Wang and G. Chen, Nat. Commun., 2018, 9, 1335.

62 P. Huber, T. Blättler, M. Textor, W. Leitenberger, U. Pietsch and T. Geue, Colloids Surf., A, 2008, 321, 113.

63 K. Vegso, M. Jergel, P. Siffalovic, M. Kotlar, Y. Halahovets, M. Hodas, M. Pelletta and E. Majkova, Sens. Actuators, A, 2016, 241, 87.

64 P. Huber, O. Bunk, U. Pietsch, M. Textor and T. Geue, J. Phys. Chem. B, 2010, 114, 12473.

65 M. A. Schroer, C. Gutt, F. Lehmkühler, B. Fischer, I. Steinke, F. Westermeier, M. Sprung and G. Grübel, Soft Matter, 2015, 11, 5465.

66 D. N. ten Napel, J.-M. Meijer and A. V. Petukhov, Appl. Sci., 2021, 11, 5117.

67 M. Quesada-Pérez, A. Moncho-Jordá, F. Martínez-López and R. Hidalgo-Álvarez, J. Chem. Phys., 2001, 115, 10897.

68 P. Batys, M. Nosek and P. Weroński, Appl. Surf. Sci., 2015, 332, 327.

69 L. Zaidouny, T. Bohlein, R. Roth and C. Bechinger, Soft Matter, 2013, 9, 9230.

70 S. C. Rödner, P. Wedin and L. Bergström, Langmuir, 2002, 18, 9327.

71 V. Senthil Kumar and V. Kumaran, J. Chem. Phys., 2005, 123, 114501.

72 V. Lotito and T. Zambelli, Nanomaterials, 2019, 9, 921.

73 D. Weaire and N. Rivier, Contemp. Phys., 1984, 25, 59.

74 I. Buttinoni, M. Steinacher, H. T. Spanke, J. Pokki, S. Bahmann, B. Nelson, G. Foffi and L. Isa, Phys. Rev. E, 2017, 95, 012610.

75 A. T. Gray, E. Mould, C. P. Royall and I. Williams, J. Phys.: Condens. Matter, 2015, 27, 194108. 
76 P. J. Steinhardt, D. R. Nelson and M. Ronchetti, Phys. Rev. B: Condens. Matter Mater. Phys., 1983, 28, 784.

77 C. A. Murray, in Bond-Orientational Order in Condensed Matter Systems, ed. K. J. Strandburg, Springer, New York, NY, 1992, p. 137.

78 P. Dillmann, G. Maret and P. Keim, J. Phys.: Condens. Matter, 2012, 24, 464118.

79 K. J. Strandburg, in Bond-Orientational Order in Condensed Matter Systems, ed. K. J. Strandburg, Springer, New York, NY, 1992, p. 32.

80 D. R. Nelson and B. I. Halperin, Phys. Rev. B: Condens. Matter Mater. Phys., 1979, 19, 2457.

81 S. Ramananarivo, E. Ducrot and J. Palacci, Nat. Commun., 2019, 10, 3380.

82 Y. Kusaka, N. Ishida and H. Ushijima, Soft Matter, 2013, 9, 3155.

83 E. Locatelli and E. Bianchi, Soft Matter, 2018, 14, 8119.

84 Z. Guo, P. Wu and J. T. Kindt, Soft Matter, 2019, 15, 1027.

85 E. Tamborini, C. P. Royall and P. Cicuta, J. Phys.: Condens. Matter, 2015, 27, 194124.

86 X. Qi, Y. Chen, Y. Jin and Y.-H. Yang, J. Korean Phys. Soc., 2006, 49, 1682.

87 P. Kumnorkaew, Y.-K. Ee, N. Tansu and J. F. Gilchrist, Langmuir, 2008, 24, 12150.

88 A. J. Armstrong, R. C. Mockler and W. J. O'Sullivan, J. Phys.: Condens. Matter, 1989, 1, 1707.

89 A. L. Thorneywork, J. L. Abbott, D. G. A. L. Aarts, P. Keim and R. P. A. Dullens, J. Phys.: Condens. Matter, 2018, 30, 104003.

90 R. Aslam, S. Ardanza-Trevijano, K. M. Poduska, A. Yethiraj and W. González-Viñas, Phys. Rev. E, 2017, 95, 032607.

91 W. Mickel, S. C. Kapfer, G. E. Schröder-Turk and K. Mecke, J. Chem. Phys., 2013, 138, 044501.

92 P. Bagheri, A. M. Almudallal, A. Yethiraj and K. M. Poduska, Langmuir, 2015, 31, 8251.

93 P. Bagheri, A. M. Almudallal, A. Yethiraj and K. M. Poduska, Langmuir, 2016, 32, 2524.

94 A. Yethiraj, A. Wouterse, B. Groh and A. van Blaaderen, Phys. Rev. Lett., 2004, 92, 058301.

95 J.-M. Meijer, V. Meester, F. Hagemans, H. N. W. Lekkerkerker, A. P. Philipse and A. V. Petukhov, Langmuir, 2019, 35, 4946.

96 M. Kramár, A. Goullet, L. Kondic and K. Mischaikow, Phys. Rev. E, 2013, 87, 042207.

97 M. Kramár, A. Goullet, L. Kondic and K. Mischaikow, Phys. Rev. E, 2014, 90, 052203.

98 M. Kramár, A. Goullet, L. Kondic and K. Mischaikow, Phys. D, 2014, 283, 37.

99 S. Ardanza-Trevijano, I. Zuriguel, R. Arévalo and D. Maza, Phys. Rev. E, 2014, 89, 052212.

100 V. Robins and K. Turner, Phys. D, 2016, 334, 99.

101 L. Malaquin, T. Kraus, H. Schmid, E. Delamarche and H. Wolf, Langmuir, 2007, 23, 11513.

102 J. Gao, S. Semlali, J. Hunel, D. Montero, Y. Battie, D. Gonzalez-Rodriguez, R. Oda, G. L. Drisko and E. Pouget, Chem. Mater., 2020, 32, 821.
103 D. Noguera-Marín, C. L. Moraila-Martínez, M. CabrerizoVílchez and M. A. Rodríguez-Valverde, Eur. Phys. J. A, 2016, 39, 20.

104 A. S. Dimitrov and K. Nagayama, Langmuir, 1996, 12, 1303.

105 G. Jing, H. Bodiguel, F. Doumenc, E. Sultan and B. Guerrier, Langmuir, 2010, 26, 2288.

106 J. J. Diao and M. G. Xia, Colloids Surf., A, 2009, 338, 167.

107 S. Watanabe, K. Inukai, S. Mizuta and M. T. Miyahara, Langmuir, 2009, 25, 7287.

108 H. Bodiguel, F. Doumenc and B. Guerrier, Langmuir, 2010, 26, 10758.

109 L. Landau and B. Levich, Acta Physicochim. URSS, 1942, $17,42$.

110 B. Derjaguin, Acta Physicochim. URSS, 1945, 20, 349.

111 S. Palma and H. Lhuissier, J. Fluid Mech., 2019, 869, R3.

112 G. Berteloot, A. Daerr, F. Lequeux and L. Limat, Chem. Eng. Process., 2013, 68, 69.

113 N. A. Fleck, R. M. McMeeking and T. Kraus, Langmuir, 2015, 31, 13655 .

114 K. W. Tan, G. Li, Y. K. Koh, Q. Yan and C. C. Wong, Langmuir, 2008, 24, 9273.

115 J. Zhang, H. Liu, Z. Wang and N. Ming, J. Appl. Phys., 2008, 103, 013517.

116 T. Alfrey, E. B. Bradford, J. W. Vanderhoff and G. Oster, J. Opt. Soc. Am., 1954, 44, 603.

117 V. N. Truskett and K. J. Stebe, Langmuir, 2003, 19, 8271.

118 L. V. Govor, G. Reiter, J. Parisi and G. H. Bauer, Phys. Rev. E, 2004, 69, 061609.

119 D. M. Kuncicky and O. D. Velev, Langmuir, 2008, 24, 1371.

120 T. P. Bigioni, X.-M. Lin, T. T. Nguyen, E. I. Corwin, T. A. Witten and H. M. Jaeger, Nat. Mater., 2006, 5, 265.

$121 \mathrm{H}$. $\mathrm{Hu}$ and R. G. Larson, in Evaporative Self-Assembly of Ordered Complex Structures, World Scientific, 2011, p. 1.

122 R. D. Deegan, O. Bakajin, T. F. Dupont, G. Huber, S. R. Nagel and T. A. Witten, Nature, 1997, 389, 827.

123 C. D. Dushkin, H. Yoshimura and K. Nagayama, Chem. Phys. Lett., 1993, 204, 455.

124 R. Bhardwaj, X. Fang, P. Somasundaran and D. Attinger, Langmuir, 2010, 26, 7833.

125 M. Majumder, C. S. Rendall, J. A. Eukel, J. Y. L. Wang, N. Behabtu, C. L. Pint, T.-Y. Liu, A. W. Orbaek, F. Mirri, J. Nam, A. R. Barron, R. H. Hauge, H. K. Schmidt and M. Pasquali, J. Phys. Chem. B, 2012, 116, 6536.

126 K. N. Al-Milaji and H. Zhao, J. Phys. Chem. C, 2019, 123, 12029.

127 A. Vital, M. Vayer, T. Tillocher, R. Dussart, M. Boufnichel and C. Sinturel, Appl. Surf. Sci., 2017, 393, 127.

128 M. H. Patel, T. K. Chaudhuri, V. K. Patel, T. Shripathi, U. Deshpande and N. P. Lalla, RSC Adv., 2017, 7, 4422.

129 G. W. Kim, E. H. Kwon, M. Kim and Y. D. Park, J. Phys. Chem. C, 2019, 123, 23255.

130 D. Quéré, A. de Ryck and O. O. Ramdane, EPL, 1997, 37, 305.

131 A. Q. Shen, B. Gleason, G. H. McKinley and H. A. Stone, Phys. Fluids, 2002, 14, 4055. 
132 R. Krechetnikov and G. M. Homsy, J. Fluid Mech., 2006, 559, 429.

133 R. Krechetnikov and G. M. Homsy, Phys. Fluids, 2005, 17, 102108.

134 J. Delacotte, L. Montel, F. Restagno, B. Scheid, B. Dollet, H. A. Stone, D. Langevin and E. Rio, Langmuir, 2012, 28, 3821.

135 M. H. Kim, H. K. Choi, O. O. Park and S. H. Im, Appl. Phys. Lett., 2006, 88, 143127.

136 K. Nagayama, Colloids Surf., A, 1996, 109, 363.

137 E. Bindini, G. Naudin, M. Faustini, D. Grosso and C. Boissière, J. Phys. Chem. C, 2017, 121, 14572.

138 G. J. A. A. Soler-Illia, P. C. Angelomé, M. C. Fuertes, D. Grosso and C. Boissiere, Nanoscale, 2012, 4, 2549.

139 L. Cademartiri, A. Sutti, G. Calestani, C. Dionigi, P. Nozar and A. Migliori, Langmuir, 2003, 19, 7944.

140 D. D. Brewer, T. Shibuta, L. Francis, S. Kumar and M. Tsapatsis, Langmuir, 2011, 27, 11660.

141 H. Ko, H.-W. Lee and J. Moon, Thin Solid Films, 2004, 447, 638.

142 E. Adachi and K. Nagayama, Langmuir, 1996, 12, 1836.

143 E. Adachi and K. Nagayama, Adv. Biophys., 1997, 34, 81.

144 Z. Yuan, D. N. Petsev, B. G. Prevo, O. D. Velev and P. Atanassov, Langmuir, 2007, 23, 5498.

145 M. Le Berre, Y. Chen and D. Baigl, Langmuir, 2009, 25, 2554.

146 C.-Y. Cai, K.-Y. A. Lin and H. Yang, Appl. Phys. Lett., 2014, 105, 201913.

147 J. Ouyang, T.-F. Guo, Y. Yang, H. Higuchi, M. Yoshioka and T. Nagatsuka, Adv. Mater., 2002, 14, 915.

148 E. Pérez-Gutiérrez, J. Lozano, J. Gaspar-Tánori, J.-L. Maldonado, B. Gómez, L. López, L.-F. Amores-Tapia, O. Barbosa-García and M.-J. Percino, Sol. Energy, 2017, 146, 79.

149 S. Matsushita, T. Miwa and A. Fujishima, Langmuir, 1997, 13, 2582.

150 B. G. Prevo and O. D. Velev, Langmuir, 2004, 20, 2099.

151 H. Yang and P. Jiang, Langmuir, 2010, 26, 13173.

152 K. Chen, S. V. Stoianov, J. Bangerter and H. D. Robinson, J. Colloid Interface Sci., 2010, 344, 315.

153 A. D. Ormonde, E. C. M. Hicks, J. Castillo and R. P. Van Duyne, Langmuir, 2004, 20, 6927.

154 M. Mittal, R. K. Niles and E. M. Furst, Nanoscale, 2010, 2, 2237.

155 P. Born, S. Blum, A. Munoz and T. Kraus, Langmuir, 2011, 27, 8621.

156 J. S. Jenkins, M. C. Flickinger and O. D. Velev, Coatings, 2013, 3, 26.

157 K. Joshi, T. Muangnapoh, M. D. Stever and J. F. Gilchrist, Langmuir, 2015, 31, 12348.

158 T. Muangnapoh, A. L. Weldon and J. F. Gilchrist, Appl. Phys. Lett., 2013, 103, 181603.

159 A. L. Weldon, PhD Thesis, Lehigh University, 2014.

160 T. Muangnapoh, PhD Thesis, Lehigh University, 2015.

161 S. Das, E. M. Duraia, O. D. Velev, J. R. Gatabi and G. W. Beall, Surf. Coat. Technol., 2017, 319, 353.
162 D. J. Lacks and J. R. Wienhoff, J. Chem. Phys., 1999, 111, 398.

163 B. Cabane, J. Li, F. Artzner, R. Botet, C. Labbez, G. Bareigts, M. Sztucki and L. Goehring, Phys. Rev. Lett., 2016, 116, 208001.

164 C. Y. Lau and W. B. Russel, AlChE J., 2014, 60, 1287.

165 A. S. Dimitrov, T. Miwa and K. Nagayama, Langmuir, 1999, 15, 5257.

166 G. B. Jeffery and L. N. G. Filon, Proc.: Math., Phys. Eng. Sci., 1922, 102, 161.

167 D. M. Kuncicky, B. G. Prevo and O. D. Velev, J. Mater. Chem., 2006, 16, 1207-1211.

168 S. Thiemann, M. Gruber, I. Lokteva, J. Hirschmann, M. Halik and J. Zaumseil, ACS Appl. Mater. Interfaces, 2013, 5, 1656.

169 R. Srikantharajah, T. Schindler, I. Landwehr, S. Romeis, T. Unruh and W. Peukert, Nanoscale, 2016, 8, 19882.

170 V. R. Dugyala and M. G. Basavaraj, RSC Adv., 2015, 5, 60079.

171 H. Shimoda, S. J. Oh, H. Z. Geng, R. J. Walker, X. B. Zhang, L. E. McNeil and O. Zhou, Adv. Mater., 2002, 14, 899.

172 M. E. Spotnitz, D. Ryan and H. A. Stone, J. Mater. Chem., 2004, 14, 1299.

173 P. Atanasova, N. Stitz, S. Sanctis, J. H. M. Maurer, R. C. Hoffmann, S. Eiben, H. Jeske, J. J. Schneider and J. Bill, Langmuir, 2015, 31, 3897.

174 J.-M. Meijer, F. Hagemans, L. Rossi, D. V. Byelov, S. I. R. Castillo, A. Snigirev, I. Snigireva, A. P. Philipse and A. V. Petukhov, Langmuir, 2012, 28, 7631.

175 C. L. Moraila-Martínez, M. A. Cabrerizo-Vílchez and M. A. Rodríguez-Valverde, Soft Matter, 2013, 9, 1664.

176 A. Askounis, K. Sefiane, V. Koutsos and M. E. R. Shanahan, Colloids Surf., A, 2014, 441, 855.

177 M. J. Pavan and R. Shenhar, J. Mater. Chem., 2011, 21, 2028.

178 N. Vogel, M. Retsch, C.-A. Fustin, A. del Campo and U. Jonas, Chem. Rev., 2015, 115, 6265.

179 O. Kletenik-Edelman, C. G. Sztrum-Vartash and E. Rabani, J. Mater. Chem., 2009, 19, 2872.

180 J. Tien, A. Terfort and G. M. Whitesides, Langmuir, 1997, 13, 5349.

181 C. von Bojnicic-Kninski, R. Popov, E. Dörsam, F. F. Loeffler, F. Breitling and A. Nesterov-Mueller, $A d v$. Funct. Mater., 2017, 27, 1703511.

182 C.-A. Fustin, G. Glasser, H. W. Spiess and U. Jonas, Adv. Mater., 2003, 15, 1025.

183 Y. Masuda, T. Itoh and K. Koumoto, Langmuir, 2005, 21, 4478.

184 Q. Guo, C. Arnoux and R. E. Palmer, Langmuir, 2001, 17, 7150.

185 W. M. Choi and O. O. Park, Colloids Surf., A, 2006, 277, 131.

186 B. Viallet, L. Ressier, L. Czornomaz and N. Decorde, Langmuir, 2010, 26, 4631. 
187 C. Belgardt, T. Blaudeck, C. von Borczyskowski and H. Graaf, Adv. Eng. Mater., 2014, 16, 1090.

188 C.-A. Fustin, G. Glasser, H. W. Spiess and U. Jonas, Langmuir, 2004, 20, 9114.

189 C. Yi, Y. Yang, B. Liu, J. He and Z. Nie, Chem. Soc. Rev., 2020, 49, 465.

190 S. B. Darling, Prog. Polym. Sci., 2007, 32, 1152.

191 M. R. Bockstaller, Y. Lapetnikov, S. Margel and E. L. Thomas, J. Am. Chem. Soc., 2003, 125, 5276.

192 R. J. Spontak, R. Shankar, M. K. Bowman, A. S. Krishnan, M. W. Hamersky, J. Samseth, M. R. Bockstaller and K. Ø. Rasmussen, Nano Lett., 2006, 6, 2115.

193 A. Horechyy, B. Nandan, N. E. Zafeiropoulos, P. Formanek, U. Oertel, N. C. Bigall, A. Eychmüller and M. Stamm, Adv. Funct. Mater., 2013, 23, 483.

194 Q. Li, J. He, E. Glogowski, X. Li, J. Wang, T. Emrick and T. P. Russell, Adv. Mater., 2008, 20, 1462.

195 V. Flauraud, M. Mastrangeli, G. D. Bernasconi, J. Butet, D. T. L. Alexander, E. Shahrabi, O. J. F. Martin and J. Brugger, Nat. Nanotechnol., 2017, 12, 73.

196 T. Kraus, L. Malaquin, H. Schmid, W. Riess, N. D. Spencer and H. Wolf, Nat. Nanotechnol., 2007, 2, 570.

197 C. Lu, H. Möhwald and A. Fery, Soft Matter, 2007, 3, 1530.

198 A. Mathur, A.-D. Brown and J. Erlebacher, Langmuir, 2006, 22, 582 .

199 S. Ni, H. Wolf and L. Isa, Langmuir, 2018, 34, 2481.

200 T. Kraus, L. Malaquin, E. Delamarche, H. Schmid, N. D. Spencer and H. Wolf, Adv. Mater., 2005, 17, 2438.

201 V. Liberman, C. Yilmaz, T. M. Bloomstein, S. Somu, Y. Echegoyen, A. Busnaina, S. G. Cann, K. E. Krohn, M. F. Marchant and M. Rothschild, Adv. Mater., 2010, 22, 4298.

202 M. Asbahi, F. Wang, Z. Dong, J. K. W. Yang and K. S. L. Chong, Nanotechnology, 2016, 27, 424001.

203 V. Sharma, D. Xia, C. C. Wong, W. C. Carter and Y.-M. Chiang, J. Mater. Res., 2011, 26, 247.

204 C. Kuemin, R. Stutz, N. D. Spencer and H. Wolf, Langmuir, 2011, 27, 6305.

205 M. Juodènas, T. Tamulevičius, J. Henzie, D. Erts and S. Tamulevičius, ACS Nano, 2019, 13, 9038.

206 M. Juodènas, D. Peckus, T. Tamulevičius, Y. Yamauchi, S. Tamulevičius and J. Henzie, ACS Photonics, 2020, 7, 3130.

207 Y. Zhou, X. Zhou, D. J. Park, K. Torabi, K. A. Brown, M. R. Jones, C. Zhang, G. C. Schatz and C. A. Mirkin, Nano Lett., 2014, 14, 2157.

208 M. Asbahi, S. Mehraeen, F. Wang, N. Yakovlev, K. S. L. Chong, J. Cao, M. C. Tan and J. K. W. Yang, Nano Lett., 2015, 15, 6066.

209 S. Ni, M. J. K. Klein, N. D. Spencer and H. Wolf, Langmuir, 2014, 30, 90 .
210 K. Sugano, K. Aiba, K. Ikegami and Y. Isono, Jpn. J. Appl. Phys., 2017, 56, 06GK01.

211 J. A. Fan, K. Bao, L. Sun, J. Bao, V. N. Manoharan, P. Nordlander and F. Capasso, Nano Lett., 2012, 12, 5318.

212 N. J. Greybush, I. Liberal, L. Malassis, J. M. Kikkawa, N. Engheta, C. B. Murray and C. R. Kagan, ACS Nano, 2017, 11, 2917.

213 S. Ni, J. Leemann, I. Buttinoni, L. Isa and H. Wolf, Sci. Adv., 2016, 2, e1501779.

214 J. C. Yang and J. Y. Park, ACS Appl. Mater. Interfaces, 2016, 8, 7381.

215 C. Lu, H. Möhwald and A. Fery, Soft Matter, 2007, 3, 1530.

216 F. Ramiro-Manzano, E. Bonet, I. Rodriguez and F. Meseguer, Langmuir, 2010, 26, 4559.

217 Y. Cui, M. T. Björk, J. A. Liddle, C. Sönnichsen, B. Boussert and A. P. Alivisatos, Nano Lett., 2004, 4, 1093.

218 J. A. Liddle, Y. Cui and P. Alivisatos, J. Vac. Sci. Technol., B, 2004, 22, 3409.

219 Y. Xia, Y. Yin, Y. Lu and J. McLellan, Adv. Funct. Mater., 2003, 13, 907.

220 M. Allard, E. H. Sargent, P. C. Lewis and E. Kumacheva, Adv. Mater., 2004, 16, 1360.

221 J. Sun, Y. Y. Li, H. Dong, P. Zhan, C. J. Tang, M. W. Zhu and Z. L. Wang, Adv. Mater., 2008, 20, 123.

222 C. Shillingford, V. Grebe, A. McMullen, J. Brujic and M. Weck, Langmuir, 2019, 35, 12205.

223 E. Kumacheva, R. K. Golding, M. Allard and E. H. Sargent, Adv. Mater., 2002, 14, 221.

224 Y. Yin, Y. Lu and Y. Xia, J. Mater. Chem., 2001, 11, 987.

225 H. Kadiri, S. Kostcheev, D. Turover, R. Salas-Montiel, K. Nomenyo, A. Gokarna and G. Lerondel, Beilstein J. Nanotechnol., 2014, 5, 1203.

226 J. P. Hoogenboom, C. Rétif, E. de Bres, M. van de Boer, A. K. van Langen-Suurling, J. Romijn and A. van Blaaderen, Nano Lett., 2004, 4, 205.

227 J. P. Hoogenboom, PhD Thesis, Universiteit Utrecht, 2002. 228 Y.-H. Ye, S. Badilescu, V.-V. Truong, P. Rochon and A. Natansohn, Appl. Phys. Lett., 2001, 79, 872.

229 M. Asbahi, K. T. P. Lim, F. Wang, H. Duan, N. Thiyagarajah, V. Ng and J. K. W. Yang, Langmuir, 2012, 28, 16782-16787.

230 N. Ostrovsky, G. Le Saux, U. Argaman, I. Chen, T. Chen, C.-H. Chang, G. Makov and M. Schvartzman, Langmuir, 2021, 37, 9098.

231 X. Huang, M. Bjork, D. C. Ratchford and J. Yeom, Langmuir, 2017, 33, 12218-12226.

232 G. L. Drisko, A. Carretero-Genevrier, M. Gich, J. Gàzquez, D. Ferrah, D. Grosso, C. Boissière, J. Rodriguez-Carvajal and C. Sanchez, Adv. Funct. Mater., 2014, 24, 5494. 